\title{
Optimal supply chain design and management over a multi-period horizon under demand uncertainty. Part I: MINLP and MILP models
}

\author{
Maria Analia Rodriguez ${ }^{1}$, Aldo R. Vecchietti ${ }^{1}$, Iiro Harjunkoski ${ }^{2}$ and Ignacio E. Grossmann ${ }^{2}$ \\ ${ }^{1}$ INGAR (CONICET-UTN), Avellaneda 3657, Santa Fe 3000, Argentina \\ ${ }^{2}$ ABB AG, Corporate Research Germany, Wallstadter Straße 59, 68526 Ladenburg, Germany \\ ${ }^{3}$ Center for Advanced Process Decision-making, Department of Chemical Engineering, Carnegie Mellon \\ University, Pittsburgh, PA 15217, USA
}

\begin{abstract}
An optimization model is proposed to redesign the supply chain of spare part delivery under demand uncertainty in a specified planning horizon. Long term decisions involve new installations, expansions and elimination of warehouses handling multiple products. It is considered which warehouses should be used as repair work-shops to store, repair and deliver used units to customers. Tactical planning includes deciding inventory, and the connection links between the supply chain nodes. At the tactical level it is determined how demand of failing units is satisfied, and whether to use new or used parts. The uncertain demand is addressed by defining the optimal amount of safety stock that guarantees certain service level at a customer plant. Due to the nonlinear nature of the original formulation, a piece-wise linearization approach is applied to obtain a tight lower bound of the optimal solution. The formulation is applied to the supply chain of electric motors.
\end{abstract}

Keywords: supply chain, demand uncertainty, inventory management, mixed integer non-linear programming

\section{Introduction}

The integration of supply chain redesign and tactical decisions such as defining inventory levels and how supply chain nodes are connected is a challenging problem that can greatly impact the financial performance of a company. Rising transportation costs are key factors in decisions about where to place factories and distribution centers, and how much inventory to store. In addition, optimal inventory management has become a major goal in order to simultaneously reduce costs and improve customer service in today's increasingly competitive business environment (Daskin, Coullard and Shen, 2002). For that reason, over the last few years, there has been an increasing interest in developing enterprise-wide optimization (EWO) models to solve problems that are broad in scope and integrate several decision levels (Grossmann, 2005). EWO involves optimizing the operations of supply, manufacturing and distribution activities of a company to reduce costs, inventories and environmental impact, and to maximize profits and responsiveness.

Given a supply chain where some plants and distribution centers are already installed, the redesign problem consist of deciding on new investments as well as eliminating installed assets that are not profitable. Considering these types of decisions as an isolated problem, without taking into account certain tactical and operational decisions, could have a negative impact on the performance of 
the supply chain. Investment decisions in a supply chain directly affect transportation and inventory costs. Therefore, an integrated approach is required to obtain a more flexible and efficient supply chain.

In the particular case of the electric motors industry, the relevance of this problem is given by some key issues. On the one hand, electric motors are expensive products, so keeping them in inventory means tying a significant amount of capital. On the other hand, a motor malfunction may block the entire production of a customer's plant, and therefore obtaining a spare motor as soon as possible is critical. The same applies to e.g. wind generators, where energy is the only product.

Another special characteristic of this type of industry is given by the type of product. Most contributions in the literature assume that products are only moved forward in the supply chain, and only the demand of new products is considered. In this case, the situation is more complex. As usual, demand can be originated by new customers or new investments at customer sites. However, in addition, motors or other units that are already in use can fail. In this case, clients require replacing the failed component. An important decision in this context is whether to replace failed parts with new units or with repaired products. The latter give rise to reverse flows since failed units must be shipped from the customers to the service centers for repair. An efficient inventory management of new and used units in the supply chain warehouses is another challenge of this problem.

Customer plants typically have tens or more different types of motors or other units in their production processes, and identical units can be used for a variety of purposes. According to the type of unit and its application, the criticality of a given unit can be very different so the time a customer can wait for a replacement is case dependent. If the time requirement is very tight, it might be necessary to have some emergency stock at the customer sites.

Taking into account that e.g. a motor demand is uncertain and that it depends on the failure rate, a responsive supply chain can only be guaranteed when an effective inventory management, as well as an appropriate distribution and storage structure are planned together. Furthermore, demand uncertainty might also have a relevant influence on the warehouse capacities. In that sense, if the plan for storage capacity does not consider demand uncertainty, it might be infeasible to provide the spare parts as required.

You and Grossmann (2010) propose an optimization model to design a multi-echelon supply chain and the associated inventory systems under demand uncertainty in the chemical industry. The original model is a Mixed Integer Non-Linear Programming problem (MINLP) with a non-convex objective function for which they develop a spatial decomposition algorithm to obtain near global optimal solutions with reasonable computational expense. The supply chain involves one product, and design decisions consider the installation of new distribution centers, but no expansions or elimination of installed warehouses are considered since the model assumes only one planning period. Our approach extends this previous work introducing new considerations regarding the particular 
industrial context for electric motors and other similar parts, and complexities from the modeling point of view and novel concepts that were not considered before.

We develop an optimization model to redesign the supply chain of spare parts or units under demand uncertainty from strategic and tactical perspectives in a planning horizon consisting of multiple periods. The main objective is to redesign an optimal supply chain for the spare parts minimizing costs and deciding where to place warehouses, which installed warehouses should be eliminated, what are the stock capacities and safety stocks required, as well as how to connect the different echelons of the supply chain in order to satisfy uncertain demand of spare parts.

The uncertain demand is addressed by defining the optimal amount of safety stock that guarantees certain service level at a customer plant. In addition, the risk-pooling effect described by Eppen (1979) is taken into account when defining inventory levels in distribution centers and customer zones. One additional consideration is given by the inclusion of lost sales costs in the objective function, which was extended from the work by Parker (1964). Due to the nonlinear and large size nature of the original formulation, apiece-wise linearization algorithms applied to obtain the optimal solution.

This article is organized as follows. In section 2, a brief literature review is presented showing different approaches to represent de demand uncertainty as well as the analysis of inventory systems under deterministic and uncertain contexts. The major objective of section 3 is to characterize and describe the problem addressed in this article, giving some details regarding the challenges, industry issues and main decisions considered. Section 4 presents the approach applied in this work to handle demand uncertainty and some new considerations related to lost sales. The model formulation is presented in section 5 while section 6 describes the solution approach. Sections 7 and 8 show results and conclusions, respectively.

\section{Literature Review}

\subsection{Previous works}

Some previous work from the literature address similar problems as the one in this paper. Daskin et al. (2002) introduce an inventory-location model in which supply chain design decisions integrate inventory considerations in order to minimize investment and logistic costs under demand uncertainty. It is assumed that the connection between plants and distribution centers is given. No limitation in storage and production capacity is considered and all delivery times from supplier to distribution centers are the same. Given these assumptions and since storage decisions at customer sites are disregarded, the inventory structure is considered as a single echelon system. A similar approach can be found in Shen et al. (2003). Extending this approach, You and Grossmann (2008) formulate an MINLP model and develop effective algorithms for large-scale instances.

Santoso et al. (2005) propose a stochastic programming model and solution algorithm for solving supply chain network design problems of realistic size. Their solution methodology integrates the 
sample average approximation (SAA) scheme, with an accelerated Benders decomposition algorithm to compute high quality solutions to large-scale stochastic supply chain design problems with a large number of scenarios. The model decides which facilities to install, and how different nodes should be linked in order to minimize investment, operating and transportation costs. However, inventory management and the associated costs are neglected. Since design decisions are assumed over one period, no expansion or elimination of processing facilities are considered. Bossert and Willems (2007) extend the guaranteed service modeling framework in order to optimize the inventory policy in a supply chain. Although they address numerous real world complexities regarding inventory management, no design decisions are considered since the supply chain configuration is defined a priori.

\subsection{Approachesto model demand uncertainty}

In order to cope with demand uncertainty, there are two main approaches to consider. The first one uses a stochastic programming model where uncertainty is considered directly using a scenario based approach (Sahinidis, 2004). Each scenario is associated with certain probability of occurrence and represents one possible realization for the uncertain parameter. In general, there are two or more stages in the decision process. In the first stage, 'here and now' decisions have to be made before the uncertain parameter realization is known. In the second stage, 'wait and see' decisions are considered which are associated with a recourse action because they can be made after the random parameter is known. The main disadvantage of this method is that the model size tends to increase rapidly with the number of scenarios considered. In addition, it is not always feasible to explicitly enumerate all possible discrete values of the uncertain parameter.

The second approach is to use chance constraint approach in which each uncertain parameter is treated as a random variable with a given probability distribution (Charnes and Cooper, 1963), which is applied in several cases to model demand uncertainty (Gupta and Maranas, 2003, You and Grossmann, 2008, Rodriguez and Vecchietti, 2011). Applying this approach the demand uncertainty is considered by specifying a demand level above the mean that must be satisfied. In this way, one strategy proposed by You and Grossmann (2008) is to define the safety stock as a decision variable in the model and a guaranteed service level to reduce the shortage in the inventories. Even though this approach does not involve scenarios, the model gives rise to non-linearities in the formulation.

Given the type of problem considered, if a two-stage stochastic approach were used, the design decision would be selected in a first stage, before demand is realized, and inventory levels would be determined after demand is known. However, since the product under consideration can be critical to a customer, the lead time that the customer has to wait until the unit is ready to be used is an important variable. In fact, the lead time accepted by the customers might be rather short when a spare part fails, so a recourse action would not be a feasible option in all cases. The second approach is 
chosen because it defines a safety stock level at customer sites and warehouses in order to guarantee the customer requirements.

\subsection{Inventory evolution vs. demand in the deterministic and the uncertain cases}

From the inventory management point of view, one traditional strategy to handle inventory is to consider a base stock policy which is also called order-up-to-level policy (Zipkin, 2000). Using this method, the inventory is reviewed in every time period, and the amount ordered is determined by the difference between the base stock level and the inventory level at the time of review. If the demand is deterministic, a constant demand rate is assumed so that in every period the same amount is ordered, which is exactly the total demand expected during the lead time period. This situation does not hold when there is uncertainty. Uncertain demand means that the exact amount that will be ordered by customers during the inventory period is not known in advance even though some probabilistic information regarding the demand can be assumed. If the inventory level is not properly planned, and the demand is greater than expected, lost sales or back-orders will take place. On the contrary, if the demand is lower than forecasted, there will be an excess in the inventory level. Figures 1-a and 1-b show different stock evolutions in time horizon, considering deterministic and uncertain demand, respectively. In our approach, we determine a minimum level of products to keep in stock (safety stock) in order to guarantee that certain demand increase will be satisfied. In this case, stock evolution is shown in Figure 1-c.

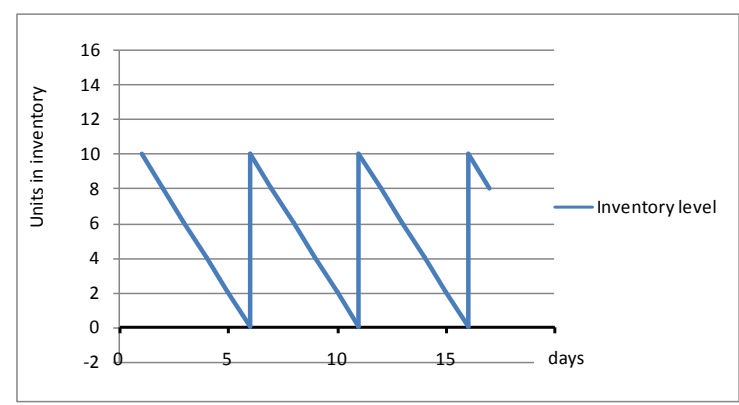

Figure 1-a. Stock evolution with deterministic demand

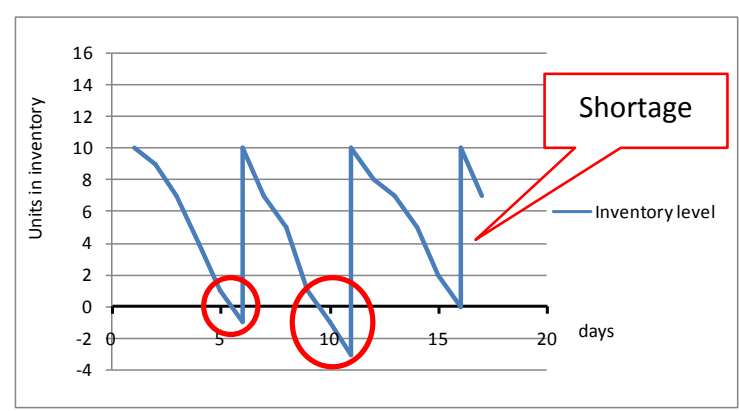

Figure 1-b. Stock evolution with uncertain demand

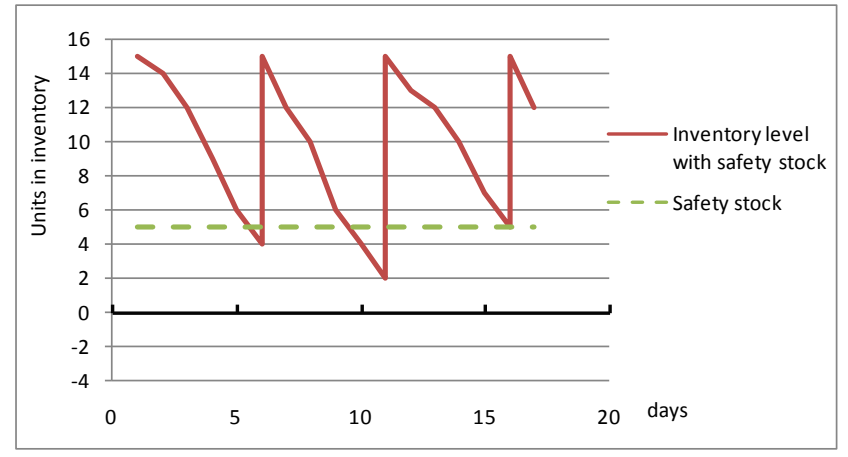

Figure 1-c. Stock evolution with uncertain demand and safety stock 


\section{Supply chain redesign in the for spare parts}

\subsection{Traditional supply chain redesign decisions}

A three echelon supply chain with a given set of factories and warehouses that produce and deliver multiple spare units to end customers is considered.

Long term decisions involve new installations, expansions, and elimination of factories and warehouses. It is also decided which warehouses should be used as repair work-shops in order to store, repair and deliver the used (repaired) units to customers. In addition, the links between factories, warehouses and end customers must be selected. We assume that several factories can provide one warehouse with the same spare part, while each end customer is assumed to be served by only one warehouse. Figure 2 represents the main design decisions in the supply chain structure, while Figure 3 shows two capacity profiles for warehouse $j_{1}$ which is fixed meaning that it is already installed at the beginning of the planning horizon, and warehouse $j_{2}$ which is installed in period 1 .

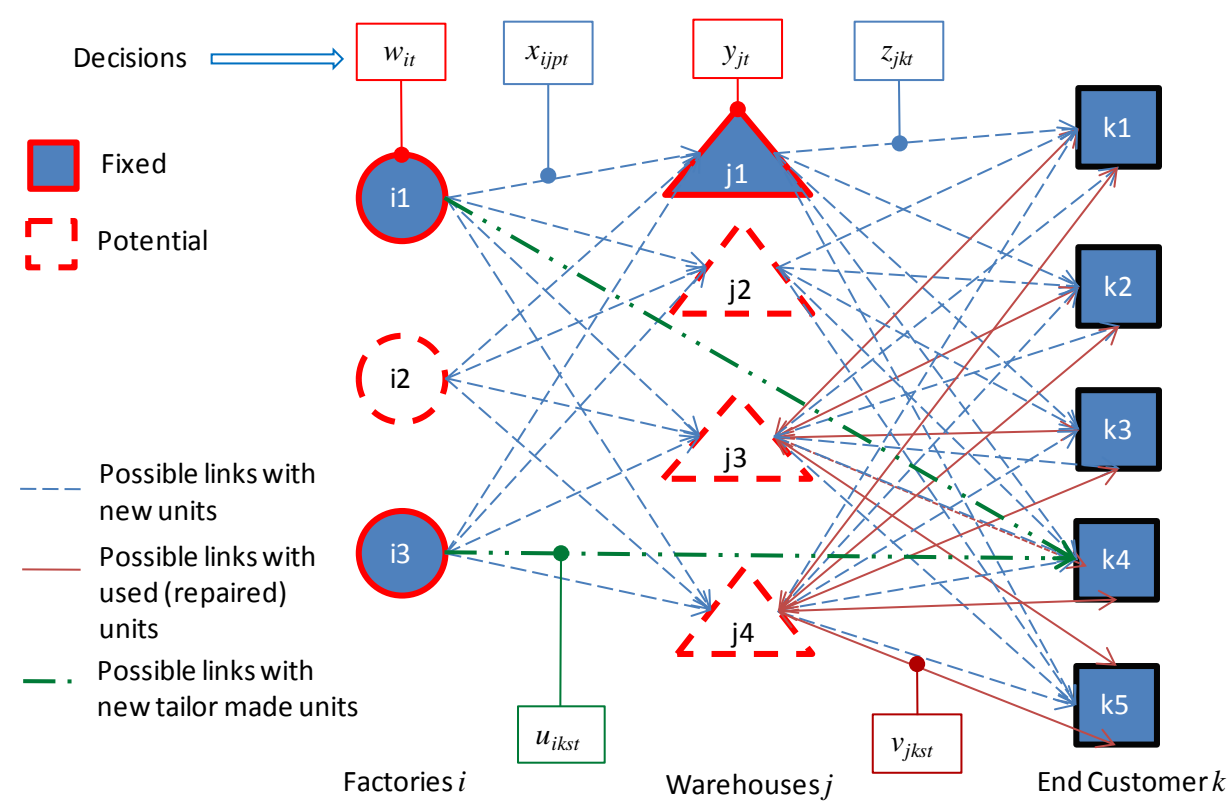

Figure 2. Supply chain design decisions
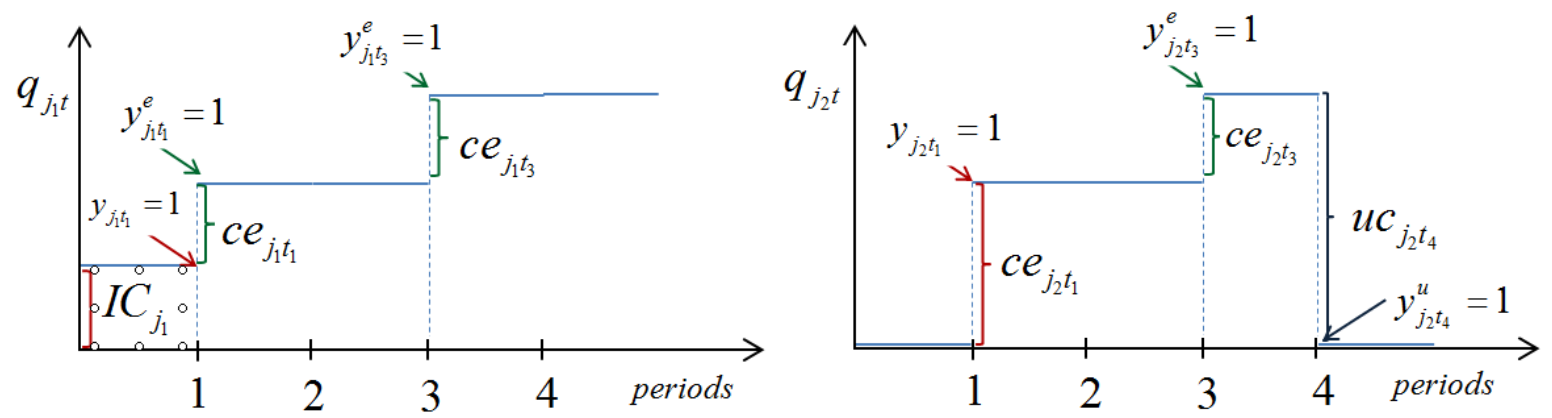

Figure 3. Comparison of capacity profiles for warehouses $j_{1}$ and $j_{2}$ where $j_{1} \in J F$ and $j_{2} \notin J F$

Figure 3 shows the following decisions for warehouses $j_{1}$ and $j_{2}$ (see Nomenclature section): 
- Warehouse $j_{1}$ is already installed in the supply chain and the initial capacity is given by $I C_{j_{1}}$. It is expanded in the first period which is indicated by binary variable $y_{j_{1} t_{1}}^{e}=1$. Capacity expansion is given by $c e_{j_{1} t_{1}}$. The same decision is made in period 3.

- Warehouse $j_{2}$ is installed in period 1 which is indicated by $y_{j_{2} t_{1}}=1$. This capacity expansion is given by $c e_{j_{2} t_{1}}$. This warehouse is expanded again in period 3. Therefore, total capacity in period 3 is given by $q_{j_{2} t_{3}}=c e_{j_{2} t_{1}}+c e_{j_{2} t_{3}}$. In period 4 this warehouse is eliminated from the supply chain, this is decided by $y_{j_{2} t_{4}}^{u}=1$ and the capacity uninstalled is given by $u c_{j_{2} t_{4}}$.

\subsection{Types of products}

Considering motors as a concrete example, these units can be separated into two types. Motors produced in factories and handled in warehouses are considered standard, i.e. they can be used for multiple purposes. In most cases, some modifications need to be applied to standard units in the warehouses in order to meet end customer requirements. After this modification the motor is considered a special one and can be shipped to the end customer. This process is shown in Figure 4 and can be also applied to other type of products.

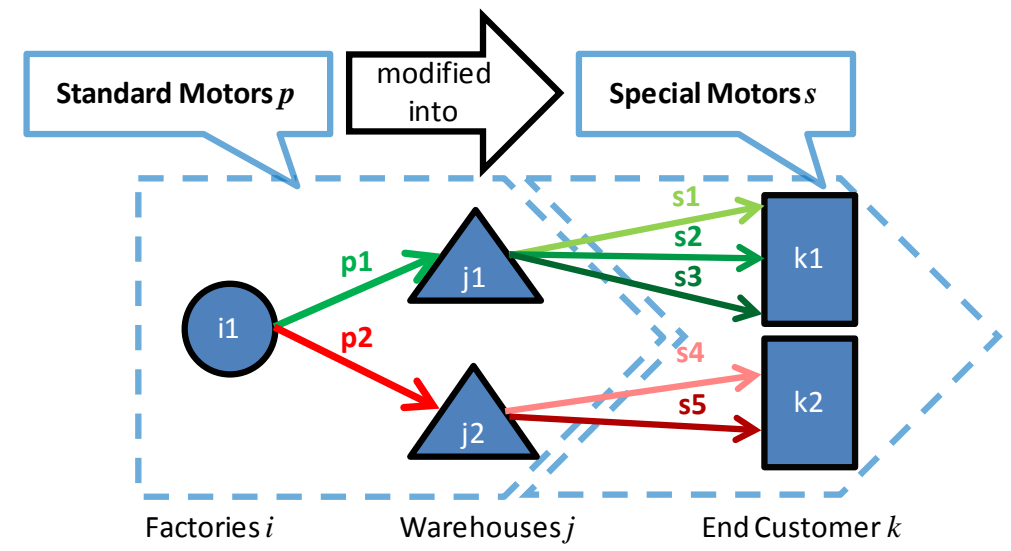

Figure 4. Supply chain structure and type of motors in each level

In addition to the previous process, tailor made parts are special units which cannot be related to the standard ones. They are directly produced at the factory according to the end customer requirements. In this case, they are delivered from the factory to the end customer, so the main decision is which factory will produce and deliver a given tailor made unit for an end customer, as shown in Figure 5. 


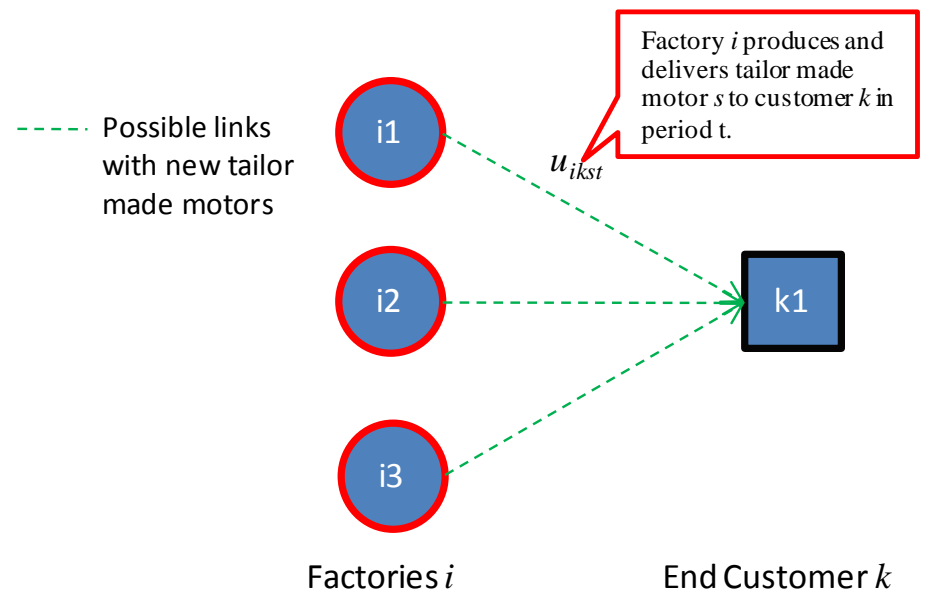

Figure 5. Tailor made motors decision

Some end customers allow that when some special units fail, they can be replaced by used (repaired) units instead of new ones. It is also assumed that some of the warehouses can act as repair workshops. As an example, Fig. 2 shows that warehouses j3 and j4 can repair spare parts and deliver them to end customers. For customers that accept used parts the model decides what part of the total demand is satisfied with new units and what part with used ones, considering an upper bound which is given by the probability that a given special unit from a given end customer can actually be repaired.

Deciding whether a customer order is satisfied with new or repaired units has also an impact on the required delivery route. Figure 6 shows that when new units are needed, the typical route is from factories to warehouses, and then from warehouses to customers. When used repaired parts are selected the route is from customers to repair workshops (which are also warehouses), and after the repair the unit goes back to the customer. Transportation costs, times and stock management are different according to the route selected.
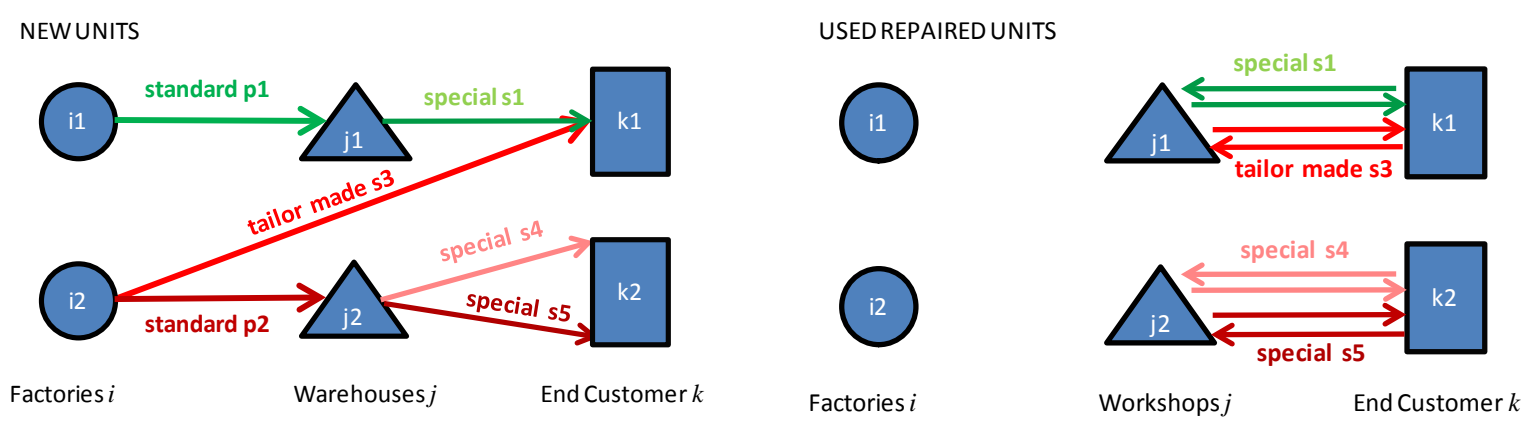

Figure 6. Spare parts route

\section{Demand uncertainty in the supply chain}

\subsection{Mean and target demand, safety stock and lost sales}

As previously mentioned, current motors or other units installed at end customers may fail during operation. Spare part demand due to failure rate is considered uncertain assuming it is a random continuous parameter with Poisson distribution, where the mean is given by the average failure rate 
$\mu^{\text {year }}$ (parts/year). As an example, let assume a spare part demand with failure distribution of mean $\mu^{\text {year }}=2000$ parts/year, which is equivalent to a daily spare part demand $(\mu)$ of 5.4795 parts/day. Since a Poisson distribution is considered, the standard deviation of the demand is given by $\sigma=\sqrt{\mu}$, which in this example is equal to 2.34. As shown in Figure 7, if the stock level is placed to satisfy the mean value of spare part demand, two possible situations could occur when spare part demand realization is known. On the one hand, if the actual spare part demand is lower than the mean forecast, unnecessary stock is held meaning an increase in inventory cost. On the other hand, if the demand is higher than the mean, lost sales or backorders take place.

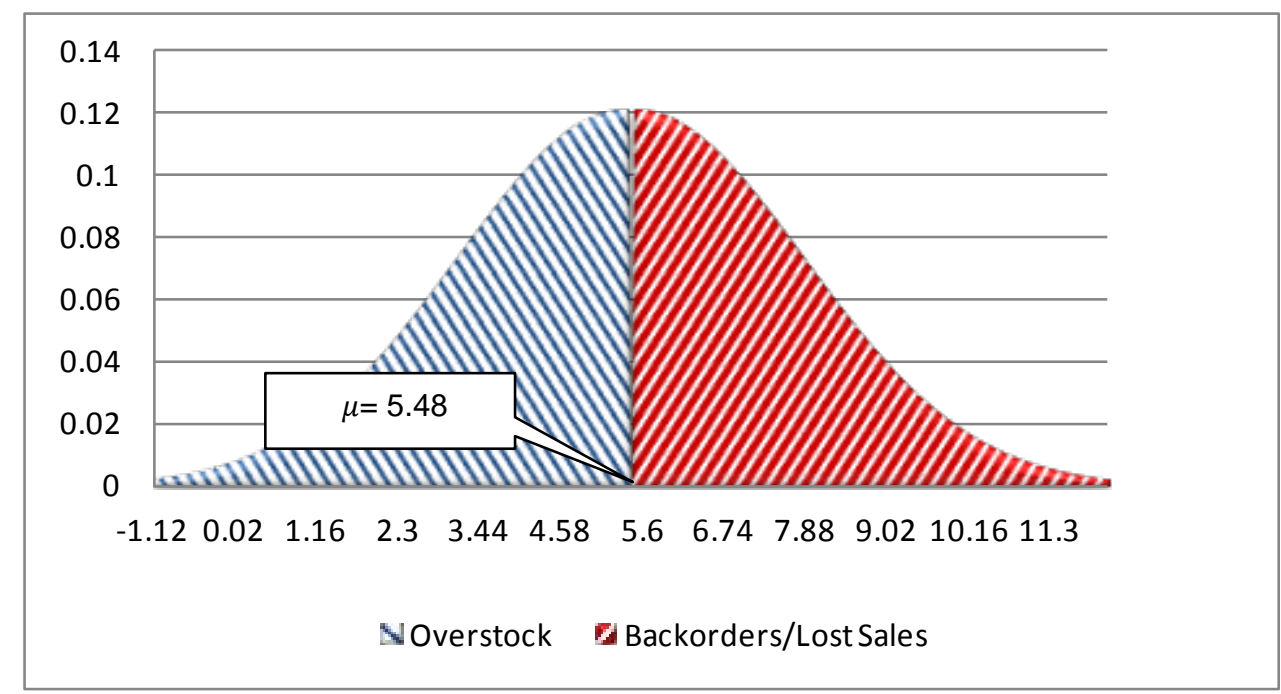

Figure 7. Demand probability distribution

If instead of simply satisfying the mean spare part demand, the supplier company is willing to satisfy some extra demand level, additional stock is needed. This safety stock is defined according to the target demand the company wants to assure and the standard deviation of the distribution. The target demand $D$ can be calculated as follows:

\section{$D=\mu+s s$; where: $\boldsymbol{\mu}$ : mean demand and ss: safety stock}

The safety stock is defined using parameter $\lambda$ which is called safety factor and represents the level of variability $\left(\sigma_{L}\right)$ the company is willing to handle. Figure 8 shows the role of the safety stock and the safety factor. The larger the safety factor is, the larger the safety stock, and as safety stock increases, fewer back-orders are expected.

$s s=\lambda \cdot \sigma_{L}$ 


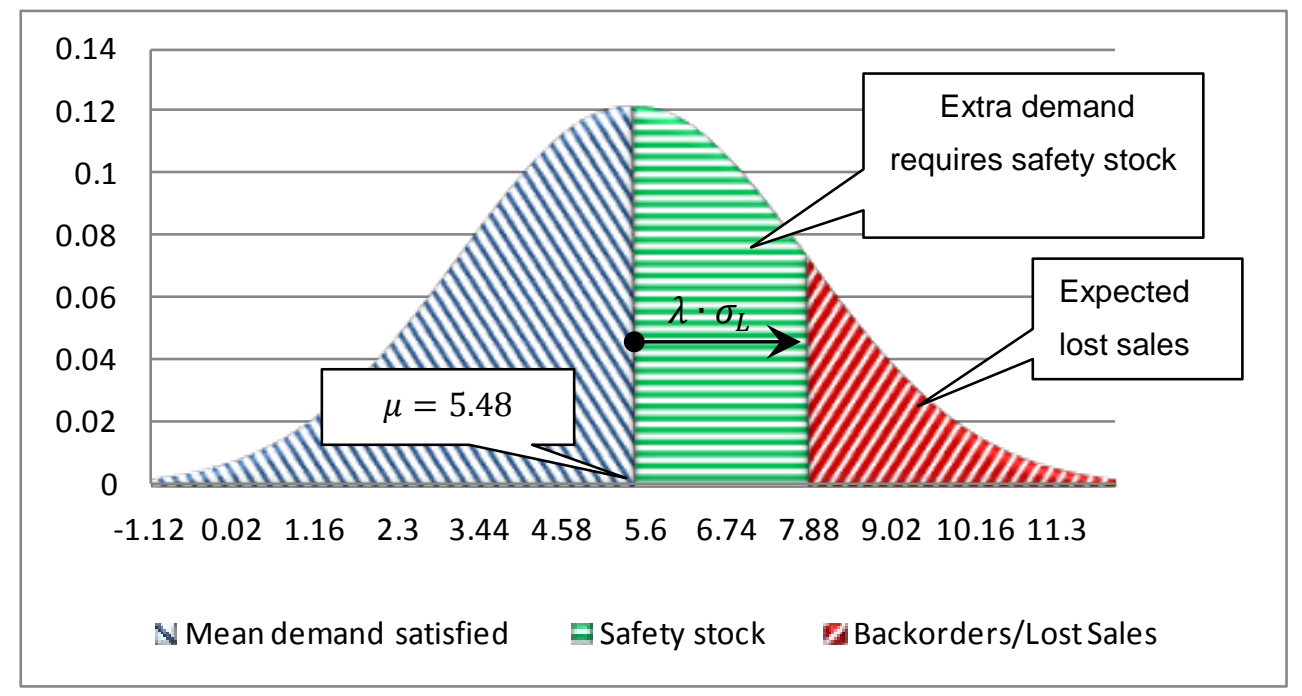

Figure 8. Demand uncertainty and safety stock

The end customers choose a service level for each unit, which is directly correlated with the maximum time the end customer expects the provider company to have a unit ready to be installed after a failure has occurred. This time is called guaranteed service time that is given in days (or a fraction of it). In order to achieve that target, in general it is necessary to have some safety stock at the end the customers' sites and warehouses.

Tactical decisions include deciding inventory levels (safety stock and expected inventory) for each type of unit at distribution centers and customer plants. Capacity constraints are also considered when planning inventory levels. At the tactical level, it is determined how the demand of failed spare parts is satisfied, and whether to use new or used spare parts.

\subsection{Guaranteed service time approach for the multi-echelon supply chain}

You and Grossmann (2010) apply the guaranteed service time approach in order to estimate the amount of safety stock in each node of the supply chain. As it was pointed out in the previous section, safety stock is calculated considering the safety factor as well as the standard deviation of demand over the lead time. When a single-stage inventory is considered this lead time is simply the inventory review period plus the replenishment time. However, when a multi-stage inventory is assumed, we need to account for the different times involved in each node of the supply chain.

The main idea of the guaranteed service time approach is that each node guarantees certain service time $T j$, in which the demand of products or materials will be satisfied. This is the maximum time the node commits to satisfying it. In the case of warehouses (internal customers), the guaranteed service time is a decision variable, while it is a parameter (external input) for end customers. In addition to this time, we also have to consider the order processing time required in each node after a new order is placed by a downstream customer. In principle, we assume that this time is independent from the order size including material handling time, transportation time from upstream node and inventory review period. 
In Fig. 9, an example of this approach is shown. The guaranteed service time by the factory is SI (parameter), and the guaranteed service time by the warehouses is $s$ (decision variable). Parameter $t_{1}$ represents the order processing time from factory $i_{1}$ to warehouse $j_{1}$.

If a new demand order is placed above the mean expected level, the warehouse must have this order ready by the guaranteed service time $s$. Considering that if the warehouse places a new order at the same time the customer order is received, it will take $t 1+S I$ units of time until the order is ready for the customer. Therefore, the net lead time defines the time span for which safety stock is required to cover demand variation. Net lead time in warehouse $j_{1}$ is shown in Figure 9.

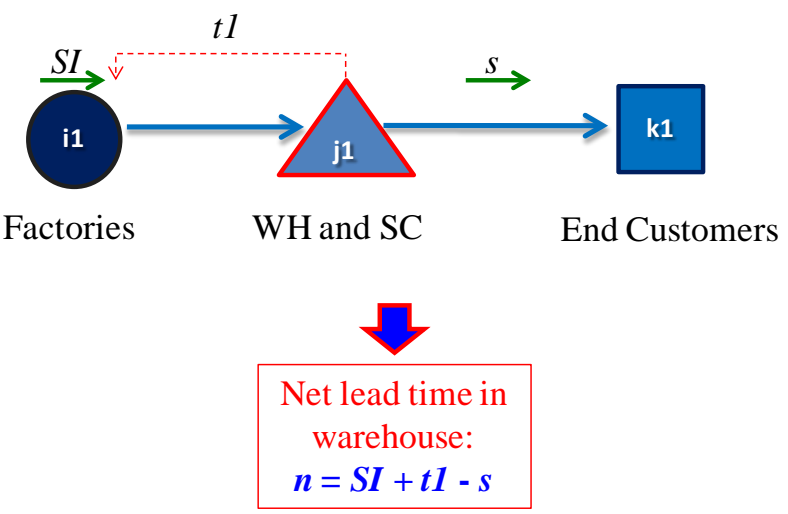

Figure 9. Net lead time in warehouse

\subsection{Expected lost sales}

The aim of this section is to introduce the incurred costs associated to lost sales. Even if safety stock is determined, demand uncertainty cannot be completely avoided. Therefore, there is always certain potential demand level that might be lost. In order to take into account this situation, we introduce the approach proposed by Parker (1964) which present a general equation for lost sales costs.

The first step is to determine the expected demand fraction which is lost in each inventory cycle, $d_{k s c t}$. Considering the nomenclature of our problem (see Nomenclature section), this equation is redefined as shown Eq. (1):

$d_{k s c t}=\sigma_{k s c t} \cdot \sqrt{l_{k s c t}} \cdot \frac{L F_{k s}}{Q_{k s c t}} \quad \forall k, \forall s, \forall c, \forall t$

where:

$\sigma_{k s c t}$ is the standard deviation of the demand of customer $\mathrm{k}$ for spare part s of criticality c in period $\mathrm{t}$

$l_{k s c t}$ is the net lead time of customer $\mathrm{k}$ for spare part $\mathrm{s}$ of criticality c in period $\mathrm{t}$

$Q_{k s c t}$ is the order size of customer $k$ for standard unit $s$ of criticality c in period $t$.

$L F_{k s}$ represents the Loss function of the demand random variable. If we estimate this function with the normal distribution, the formula of $L F_{k s}$ is given by Eq. (3). 
$L F_{k s}=\int_{\lambda 2_{k s}}^{\infty}\left(x-\lambda 2_{k s}\right) \cdot p(x) d x$

$\forall k, \forall s$

where $\lambda 2_{k s}$ is the safety factor (which limits the safety stock as well as the lost sales cost as shown in Figure 10) and $p(x)$ is the normal probability function (mean zero and standard deviation 1).

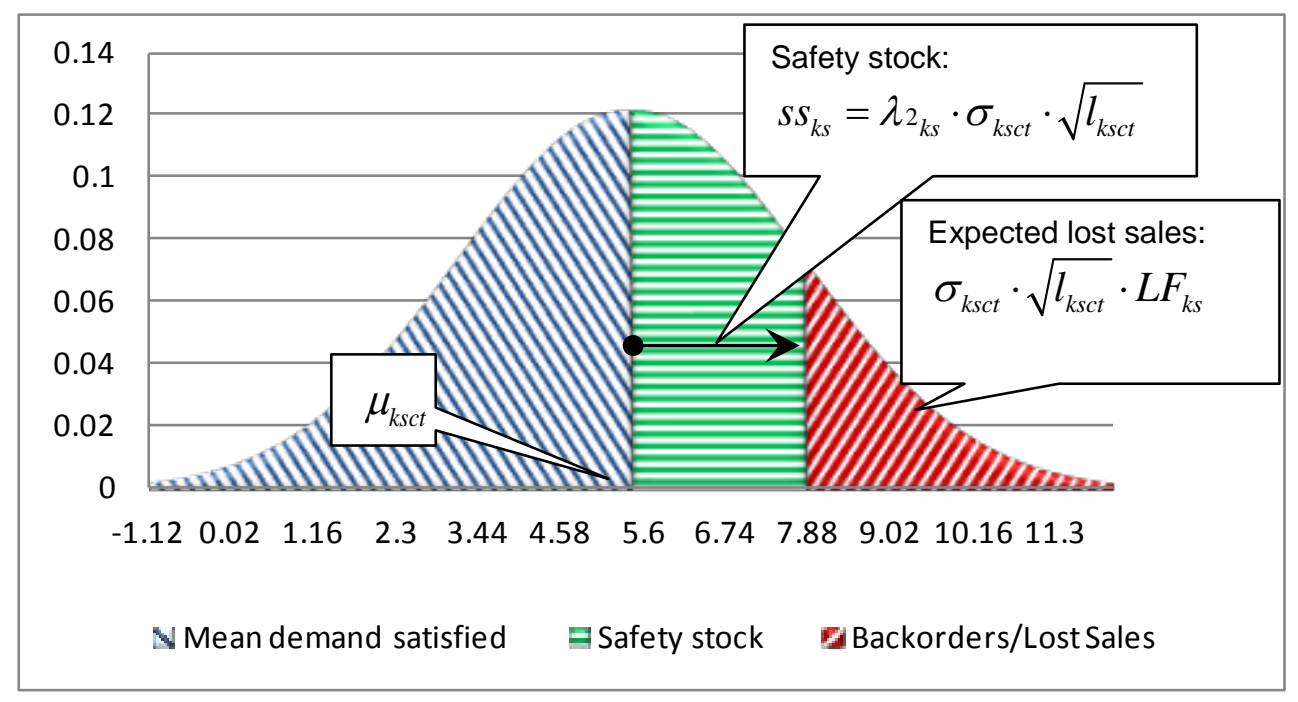

Figure 10. Demand uncertainty, safety stock and lost sales

Parker (1964) estimates the loss function, as presented in Eq. (4):

$L F_{k s}=\gamma \cdot e^{\lambda 2_{k s} / \delta} \quad \forall k, \forall s$

Parameters are determined by Parker (1964) as follows: $\gamma=0.45$ y $\delta=-0.59$.

According to Parker (1964), the annual cost due to lost sales $\left(C B_{k s c t}\right)$ for a given customer $k$ and special spare part $s$ is defined by Eq. (4):

$C B_{k s c t}=b 1_{k s} \cdot d_{k s c t} \cdot \mu_{k s c t} \cdot \chi \quad \forall k, \forall s, \forall c, \forall t$

where:

$\mu_{k s c t}$ corresponds to the daily demand of spare part $s$ of criticality $c$ from customer $k$ in period $t$ $\chi$ are the days per period $t$

$b 1_{k s}$ is the lost sales unit cost for customer $k$ and unit $s$

Then, $d_{k s t} \cdot \mu_{k s c t} \cdot \chi$ represents the quantity of lost sales in the year $t$ for the customer $k$, special spare part s of criticality $c$.

Replacing $L F_{k s}$ and $d_{k s c t}$ into $C B_{k s c t}$, the annual costs due to lost sales are given by Eq. (5),

$C B_{k s c t}=b 1_{k s} \cdot \sigma_{k s c t} \cdot \sqrt{l_{k s c t}} \cdot \frac{0.45 \cdot e^{\lambda 2_{k s /}-0.59}}{Q_{k s c t}} \cdot \mu_{k s c t} \cdot \chi \forall k, \forall s, \forall c, \forall t$

Note that:

- $b 1_{k s}$ is a parameter which is the unit cost (penalty) for each unit lost of special spare part s from customer $\mathrm{k}$.

- $\sigma_{k s c t} \cdot \sqrt{l_{k s c t}} \cdot 0.45 \cdot e^{\lambda 2_{k s} /}-0.59$ represents expected lost for special spare part s of criticality c from customer $\mathrm{k}$ in each inventory cycle of period $\mathrm{t}$. 
While:

- $\frac{\mu_{k s c t} \cdot \chi}{Q_{k s c t}}$ represents the number of cycles in the year.

Even though the safety factor $\lambda 2_{k s}$ is fixed (meaning that it is a parameter in the formulation), since standard deviation during the net lead time is a variable $\left(\sigma_{L T}=\sigma_{k s c t} \cdot \sqrt{l_{k s c t}}\right)$, the cost of lost sales is a variable as well. However, there is a difference between our model and the approach proposed by Parker (1964) because the number of cycles per year is not given by $\frac{\mu_{k s c t} \cdot \chi}{Q_{k s c t}}$, mainly for two reasons:

- First, the order size $Q_{k s c t}$ is not a variable in our problem.

- Second, the inventory policy assumed is the periodic-review (order-up-to policy, base stock level policy), which means that no equal amount is ordered in each cycle $Q_{k s c t}$. On the contrary, in each cycle the quantity ordered depends on the inventory position at the time the order is placed.

Therefore, we propose to consider the number of cycles as follows:

$$
\frac{\chi}{t 2_{j k t}} \cdot z_{j k t} \quad \forall j, \forall k, \forall(p, s) \in P S_{p s}, \forall t
$$

where $\chi$ indicates the number of days in the year, $t 2_{j k t}$ indicates the total processing time (in days) for a given standard spare part $p$ in customer site $k$ if the spare part is provided by warehouse $j$ and $Z_{j k t}$ is a binary variable which is one if warehouse $j$ provides spare parts to customer $k$ in period $t$. This formula gives us the number of cycles per year.

Therefore, the annual cost of lost sales is given by Eq. (7).

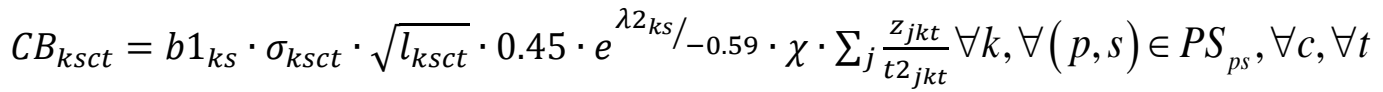

\section{Model formulation}

\subsection{MINLP multi-period problem}

Given the supply chain structure presented in Figure 2, the following equations are applied to solve the redesign problem. First, every customer $k$ must be served by one warehouse $j$ in each period $t$ according to Eq. (8).

$\sum_{j} z_{j k t}=1 \quad \forall k, \forall t$

Regarding used repaired units, at most one repair work-shop $j$ can be selected to repair unit $s$ from customer $k$ in each period $t$, as shown in Eq. (9).

$\sum_{j \in S C} v_{j k s t} \leq 1 \quad \forall k, \forall s, \forall t$

As shown in Figure 5, tailor made units are directly supplied from factories to customers. Equation (10) determines that only one factory $i$ must produce and deliver tailor made spare parts $s$ to each customer $k$ in period $t$. 
$\sum_{i} u_{i k s t}=1$

$\forall k, \forall s, \forall t$

Equation (11) determines that standard spare part $p$ can be delivered from factory $i$ to warehouse $j$ in period $t$ if the warehouse was installed and never uninstalled in period $t$ or before.

$x_{i j p t} \leq \sum_{t^{\prime} \leq t} y_{j t^{\prime}}-\sum_{t^{\prime} \leq t} y_{j t^{\prime}}^{u} \quad \forall i, \forall j, \forall p, \forall t$

Equation (12) determines that standard spare part $p$ can be delivered from factory $i$ to warehouse $j$ in period $t$ if the factory $i$ was installed and never uninstalled in period $t$ or before.

$x_{i j p t} \leq \sum_{t^{\prime} \leq t} w_{i t^{\prime}}-\sum_{t^{\prime} \leq t} w_{i t^{\prime}}^{u} \quad \forall i, \forall j, \forall p, \forall t$

Equation (13) determines that tailor made unit $s$ can be delivered from factory $i$ to end customer $k$ in period $t$ if the factory $i$ was installed and never uninstalled in period $t$ or before.

$u_{i k s t} \leq \sum_{t \prime \leq t} w_{i t},-\sum_{t \prime \leq t} w_{i t \prime}^{u} \quad \forall i, \forall(k, s) \in K T_{k s}, \forall t$

According to Eq. (14), warehouse $j$ in period $t$ can be expanded if the warehouse was previously installed.

$y_{j t}^{e} \leq \sum_{t \prime \leq t} y_{j t \prime} \quad \forall j, \forall t$

Warehouse $j$ in period $t$ can be eliminated or uninstalled in Eq. (15) if the warehouse was previously installed.

$y_{j t}^{u} \leq \sum_{t \prime \leq t} y_{j t,} \quad \forall j, \forall t$

Equation(16) establishes that factory $i$ in period $t$ can be expanded if the factory was previously installed.

$w_{i t}^{e} \leq \sum_{t \prime \leq t} w_{i t \prime} \quad \forall i, \forall t$

According to Eq. (17), factory $i$ in period $t$ can be eliminated or uninstalled if that factory was previously installed.

$w_{i t}^{u} \leq \sum_{t \prime \leq t} w_{i t \prime} \quad \forall i, \forall t$

As shown in Eq. (18), a customer $k$ can be served by a warehouse $j$ in period $t$ if that warehouse has been previously installed, and was never uninstalled before and during that period.

$z_{j k t} \leq \sum_{t \prime \leq t} y_{j t}-\sum_{t \prime \leq t} y_{j t \prime}^{u} \quad \forall j, \forall k, \forall t$

Similar to the previous constraint, Eq. (19)allows that a repair work-shop serves a customer $k$ with used units $s$ in period $t$ if that work-shop has been previously installed, and was never uninstalled before and during that period.

$v_{j k s t} \leq \sum_{t \prime \leq t} y_{j t^{\prime}}-\sum_{t \prime \leq t} y_{j t \prime}^{u} \quad \forall j \in S C, \forall k, \forall s, \forall t$

As it was previously mentioned, demand of units due to failure rate can be satisfied with new and repaired used units. Constraint (20) establishes that total mean demand $\mu_{k s c t}$ must be satisfied either with new ( $\left.\mu_{i j k p t}^{\text {new }}\right)$ or with used parts ( $\left.\mu_{j k s t}^{\text {used }}\right)$.

$\sum_{i} \sum_{j} \mu_{i j k p t}^{n e w}+\sum_{j \in S C} \sum_{\substack{s \in\left(K S C_{k s c} \cap P S_{p s} \cap C T_{k s}\right) \\ s \notin K T_{k s}}} \sum_{j} \mu_{j k s t}^{u s e d}=\sum_{\substack{s \in P S_{p s} \\ s \notin K T_{k s}}} \sum_{c \in K S C_{k s c}} \mu_{k s c t} \forall k, \forall p, \forall t$ 
In case the customer does not allow repaired parts, Eq. (21) establishes that the total demand must be satisfied with new units. Variable $\mu_{i j k p t}^{\text {new }}$ is needed in order to determine from which factory $i$ and warehouse $j$ demand of customer $k$ in period $t$ is satisfied.

$$
\sum_{i} \sum_{j} \mu_{i j k p t}^{\text {new }}=\sum_{\substack{s \in P S_{p s} \\ s \notin\left(K T_{k s} \cup C T_{k s}\right)}} \sum_{c \in K S C_{k s c}} \mu_{k s c t} \quad \forall k, \forall p, \forall t
$$

In the case of tailor made parts, Eq. (22)is applied. The total demand for tailor made parts must be satisfied either with new or used units. The main difference between standard and tailor made units regarding model formulation is that tailor made spare parts are delivered directly from plants, while standard spare parts are delivered from warehouses.

$$
\sum_{i} \tau_{i k s t}^{n e w}+\sum_{j \epsilon S C} \tau_{j k s t}^{u s e d}=\sum_{c \in K S C_{k s c}} \mu_{k s c t} \quad \forall(k, s) \in K T_{k s}, \forall t
$$

In the case that the customer does not allow repaired spare parts to satisfy tailor made units demand, Eq. (23) determines that all units expected to fail are replaced by new units.

$$
\sum_{i} \tau_{i k s t}^{n e w}=\sum_{c \in K S C_{k s c}} \mu_{k s c t} \quad \forall(k, s) \notin\left(K T_{k s} \cup C T_{k s}\right)
$$

Eq. (24)is a bilinear inequality that represents an upper bound for variable $\mu_{i j k p t}^{\text {new }}$, where binary variables $X_{i j p t}$ and $z_{j k t}$ represent the selected links in the supply chain. The first variable is one if factory $i$ produces and delivers standard unit $p$ to warehouse $j$ in period $t$, while the second is one if warehouse $j$ delivers units to end customer $k$ in period $t$. Only if both variables are positive, then demand of customer $k$ can be satisfied with new units.

$$
\mu_{i j k p t}^{n e w} \leq \sum_{\substack{s \in P S_{p s} \\ s \notin K T_{k s}}} \sum_{c \in K S C_{k s c}} \mu_{k s c t} \cdot x_{i j p t} \cdot z_{j k t} \quad \forall i, \forall j, \forall k, \forall p, \forall t
$$

Eq. (25) has the same purpose as Eq. (24), it is an upper bound for mean demand level in each node of the supply chain according to the selected links, in this case, for tailor made units.

$\tau_{i k s t}^{\text {new }} \leq \sum_{c \in K S C_{k s c}} \mu_{k s c t} \cdot u_{i k s t} \quad \forall i, \forall(k, s) \in K T_{k s}, \forall t$

Eq. (26) determines that if repair workshop j (also warehouse j) is selected to repair special unit $s$ of end customer $k$ in period $t$, given by binary variable $v_{j k s t}$, then the total amount of used spare parts required is given by the expected demand level $\mu_{k s c t}$ multiplied by the repairing probability $r p_{k s}$.

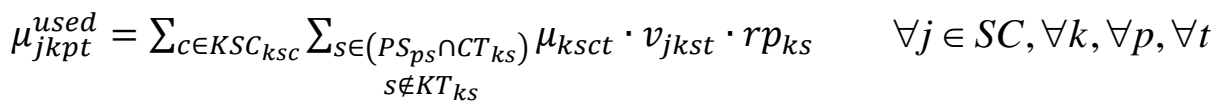

Some customers might also allow tailor made spare parts to be repaired. If that is the case, the total amount of units satisfied with used repaired units is given by the total demand due to failure rate multiplied by repairing probability. This constraint is given in Eq. (27).

$$
\tau_{j k s t}^{u s e d}=\sum_{c \in K S C_{k s c}} \mu_{k s c t} \cdot v_{j k s t} \cdot r p_{k s} \quad \forall j \in S C, \forall(k, s) \in K T_{k s}, \forall t
$$

The net lead time of warehouse $j$, standard unit $p$ in period $t$ is determined in Eq. (28). As mentioned, a safety stock level is defined in order to prevent a shortage in stock due to uncertain 
demand increase. As explained in section 4.2, this safety stock level is calculated according to the net lead time determined in Eq. (28).

$n_{j p t} \geq\left(S I_{i p}+t 1_{i j p}\right) \cdot x_{i j p t}-s_{j p t} \quad \forall i, \forall j, \forall p, \forall t$

Net lead time of tailor made unit $s$ and customer $k$ in period $t$ is calculated in Eq. (29). Similarly, the net lead time of special spare parts $s$ for each $k$ in period $t$ is determined in Eq. (30). While Eq. (29) is linear, Eq. (30) involves a bilinear product of variable $S_{j p t}$ by $Z_{j k t}$.

$$
\begin{array}{lr}
m_{k s c t} \geq\left(G I_{i s}+t 3_{i k s}\right) \cdot u_{i k s t}-R_{k s c} & \forall i, \forall(k, s, c) \in\left(K S C_{k s c} \cap K T_{k s}\right), \forall t \\
l_{k s c t} \geq \sum_{j} s_{j p t} \cdot z_{j k t}+\sum_{j} t 2_{j k p} \cdot z_{j k t}-R_{k s c} & \forall i, \forall(k, s, c) \in K S C_{k s c}, \forall(k, s, c) \notin K T_{k s}, \forall t
\end{array}
$$

The safety stock of standard spare part $p$ in warehouse $j$ for each period $t$ is determined by Eq. (31). This variable is calculated multiplying the safety factor $\lambda_{j p}$, standard deviation $\sigma_{k s c t}$ and the square root of the net lead time of warehouse $j$, standard spare part $p$ in period $t$.

$$
s s_{j p t}=\lambda_{j p} \sqrt{\sum_{k} \sum_{\substack{s \in P S_{p s} \\ s \notin K T_{k s}}} \sum_{c \in K S C_{k s c}} n_{j p t} \cdot z_{j k t} \cdot \sigma_{k s c t}{ }^{2}} \quad \forall j, \forall p, \forall t
$$

As shown in Fig. 3, the capacity profile evolves during the planning horizon according to investment decisions. In the case of warehouses, this variable is established in Eq. (32) as the capacity level in the previous period, plus the expansion $c e_{j t}$ in the present period minus the uninstalled capacity $u c_{j t}$ in period $t$. It should be noted that expansions and elimination of warehouses cannot be made simultaneously as shown later in Eq. (39). Note that when $t=1, q_{j t-1}=I C_{j}$.

$$
q_{j t}=q_{j t-1}+c e_{j t}-u c_{j t} \quad \forall j, \forall t \text {, where } q_{j 0}=I C_{j}
$$

Equation (33) determines that the maximum capacity expansion of warehouse $j$ in each period $t$, $c e_{j t}$, is given by $Q D C_{j}^{U P}$ when warehouse $j$ is installed $\left(y_{j t}=1\right.$ ) or expanded $\left(y_{j t}^{e}=1\right)$.

$c e_{j t} \leq Q D C_{j}^{U P} \cdot\left(y_{j t}+y_{j t}^{e}\right) \quad \forall j, \forall t$

In case a warehouse is already installed in period 1, Eq. (34) determines that the maximum capacity expansion of warehouse $j, c e_{j t}$, is given by $Q D C_{j}^{U P}$ when warehouse $j$ is expanded $\left(y_{j t}^{e}=1\right.$ ) .

$$
c e_{j t} \leq Q D C_{j}^{U P} \cdot y_{j t}^{e} \quad \forall j \in J F, t=1
$$

Maximum capacity in each period for warehouse $j$ is given by Eq. (35). This upper bound is given by the initial capacity, $I C_{j}$, plus the maximum capacity expansion per period, $Q D C_{j}^{U P}$, multiplied by the number of periods $t$. Note also that if this warehouse is eliminated $\left(y_{j t}^{u}=1\right)$, then capacity $q_{j t}=0$.

$q_{j t} \leq\left(Q D C_{j}^{U P} \cdot t+I C_{j}\right) \cdot\left(1-y_{j t}^{u}\right) \quad \forall j, \forall t$ 
Similarly, Eq. (36) determines an upper bound for the capacity that is uninstalled if warehouse $j$ is eliminated.

$u c_{j t} \leq\left(Q D C_{j}^{U P} \cdot t+I C_{j}\right) \cdot y_{j t}^{u} \quad \forall j, \forall t$

According to Eq. (37), warehouse $j$ can be installed only once in the time horizon.

$\sum_{t} y_{j t} \leq 1 \quad \forall j$

Also, warehouse $j$ can be uninstalled only once in the time horizon determined by Eq. (38).

$\sum_{t} y_{j t}^{u} \leq 1$

$\forall j$

Equations(39)and (40) establish that a warehouse $j$ cannot be installed, uninstalled and expanded in the same period. Only one decision at a time can be made. This constraint is applied for all warehouses $j$ that are not installed in the supply chain in period 1 (Eq. (39)), or for warehouses $j$ in any period greater than 1 (Eq. (40)).

$y_{j t}+y_{j t}^{u}+y_{j t}^{e} \leq 1$

$\forall j \notin J F, t=1$

$y_{j t}+y_{j t}^{u}+y_{j t}^{e} \leq 1$

$\forall j, \forall t>1$

Equation (41) establishes that a warehouse $j$ cannot be uninstalled and expanded in period 1 if the warehouse is already installed in the supply chain $(j \in J F)$. Only one decision at a time can be made.

$y_{j t}^{u}+y_{j t}^{e} \leq 1$

$$
\forall j \in J F, t=1
$$

Capacity of factory $i$ is defined in Eq. (42) as the capacity in the previous periods plus the capacity expansion minus capacity elimination in the same period. Note that for $t=1, q f_{i t-1}=I C F_{i}$.

$q f_{i t}=q f_{i t-1}+c e f_{i t}-u c f_{i t} \quad \forall i, \forall t$, where $q f_{i 0}=I C F_{i}$

Equation (43) determines that the maximum capacity expansion of factory $i$ in each period $t, c e f_{i t}$, is given by $Q P_{i}^{U P}$ when factory $i$ is installed ( $w_{i t}=1$ ) or expanded ( $w_{i t}^{e}=1$ ).

$c e f_{i t} \leq Q P_{i}^{U P} \cdot\left(w_{i t}+w_{i t}^{e}\right) \quad \forall i, \forall t$

If the factory is fixed at the beginning of the horizon planning ( $\forall i \in I F$ ), Eq. (44) determines that the maximum capacity expansion of factory $i$ in period $1, c e f_{i 1}$, is given by $Q P_{i}^{U P}$ only if $i$ is expanded in that period $\left(w_{i t}^{e}=1\right)$.

$c e f_{i t} \leq Q P_{i}^{U P} \cdot w_{i t}^{e} \quad \forall i \in I F, t=1$

Eq. (45) defines the maximum capacity in each period for factory $i$. This upper bound is given by the initial capacity, $I C F_{i}$, plus the maximum capacity expansion per period, $Q P_{i}^{U P}$, multiplied by the number of periods $t$. Note also that if this factory is eliminated ( $w_{i t}^{u}=1$ ), then capacity $q f_{i t}$ is set to 0 . $q f_{i t} \leq\left(Q P_{i}^{U P} \cdot t+I C F_{i}\right) \cdot\left(1-w_{i t}^{u}\right) \quad \forall i, \forall t$ 
Similarly, Eq. (46) determines an upper bound for the capacity that is uninstalled when factory $i$ is eliminated.

$u c f_{i t} \leq\left(Q P_{i}^{U P} \cdot t+I C F_{i}\right) \cdot w_{i t}^{u} \quad \forall i, \forall t$

Factory $i$ can be installed only once in the time horizon given by Eq. (47).

$\sum_{t} w_{i t} \leq 1$

$\forall i$

Also, factory $i$ can be uninstalled only once in the time horizon according to Eq. (48).

$\sum_{t} w_{i t}^{u} \leq 1$

$\forall i$

Equations (49) and (50) establish that only one decision regarding installation, elimination or expansion can be made in each period for a factory $i$. This constraint is applied for all factories $i$ which are not installed in the supply chain in period 1 (Eq. (49)) or for any factory $\mathrm{i}$ in any period greater than 1 (Eq. (50)).

$w_{i t}+w_{i t}^{u}+w_{i t}^{e} \leq 1$

$$
\begin{gathered}
\forall i \notin I F, t=1 \\
\forall i, \forall t>1
\end{gathered}
$$

Equation (51) establishes that a factory $i$ that is already installed in the supply chain ( $i \in I F)$ can be uninstalled or expanded in period 1 but only one of these decisions can be made.

$w_{i t}^{u}+w_{i t}^{e} \leq 1 \quad \forall i \in I F, t=1$

Equation (52) determines that the maximum amount of stock-keeping units (SKU) in a warehouse $j$ in period $t$ cannot exceed the capacity $q_{j t}$ which is the total amount of units that can be stored in the warehouse $j$. This amount is calculated considering both pipeline inventory and safety stock. In order to calculate the amount of new units in stock due to mean level inventory (Little, 1961), the daily demand $\mu_{i j k p t}^{\text {new }}$ is multiplied by the processing time $t 1_{i j p}$ in warehouse $j$ if the unit is delivered from factory $i$. This amount is then multiplied by a size parameter $\beta_{p}$ indicating the portion of stock capacity a given standard unit $p$ uses when it is stored. Similarly, in the case of used spare parts, the amount of them in stock is calculated as the product of the daily demand satisfied with used spare parts ( $\mu_{j k p t}^{\text {used }}$ in the case of standard spare parts and $\tau_{j k s t}^{\text {used }}$ in the case of tailor made units) multiplied by the time they are stored in average in the warehouse before they go back to the customers, $t s u_{j p}$ and $t t u_{j s}$, respectively. Both quantities are then multiplied by the corresponding size factors $\left(\beta_{p}\right.$ in the case of standard units and $\beta 2_{s}$ for tailor made). The portion of capacity used by the safety stock is calculated in the last term multiplying the safety stock $s s_{j p t}$ (in units of spare parts) by the size factor $\beta_{p}$.

$\sum_{k} \sum_{p}\left(\sum_{i} \mu_{i j k p t}^{n e w} \cdot t 1_{i j p}+\mu_{j k p t}^{u s e d} \cdot t s u_{j p}\right) \cdot \beta_{p}+\sum_{k} \sum_{s \in\left(K T_{k s} \cap C T_{k s}\right)} \tau_{j k s t}^{u s e d} \cdot t t u_{j s} \cdot \beta 2_{s}+\sum_{p} s s_{j p t} \cdot$

$\beta_{p} \leq q_{j t} \quad \forall j, \forall t$ 
Eq. (53)determines that daily demand satisfied with new units $\mu_{i j k p t}^{\text {new }}$ multiplied by capacity factor $\alpha_{p}$ cannot exceed the capacity of this factory.

$\sum_{k} \sum_{p} \sum_{j} \mu_{i j k p t}^{n e w} \cdot \alpha_{p} \leq q f_{i t} \quad \forall i, \forall t$

The following equations, from Eq.(54) to (71), introduce the different cost terms used in the objective function.

Equation (54) indicates total investment cost in new warehouses for each period, $\mathrm{TI}_{t}$. It should be noted that if a warehouse is already installed ( $j \in F J$ ) at the beginning of the horizon planning then $f_{j}$ is zero.

$T I_{t}=\sum_{j} f_{j} \cdot y_{j t} \quad \forall t$

Similarly, Eq.(55) indicates total investment cost per period in new factories, TPI . If a factory is already installed ( $i \in F I)$ at the beginning of the horizon planning then $f p_{i}$ is zero.

$T P I_{t}=\sum_{i} f p_{i} \cdot w_{i t} \quad \forall t$

Total operational fixed costs $T O F_{t}$ are given by Eq. (56). This fixed cost $o f c_{j}$ must be paid while a warehouse is installed, from the moment it is installed until it is eliminated. Therefore, if a warehouse was uninstalled in any previous or present period this cost is no longer paid. This cost will prevent to keep opened a warehouse which is not used.

TOF $_{t}=\sum_{j}$ of $c_{j} \cdot\left(\sum_{t \prime \leq t} y_{j t}-\sum_{t^{\prime} \leq t} y_{j t}^{u}\right) \quad \forall t$

Similar to the case of warehouses, there is an operational fixed cost for factories. The total costs $T P F_{t}$ is considered in Eq. (57). This fixed cost $p f c_{i}$ must be paid while the factory is installed, from the moment it is installed until it is eliminated. The aim of this cost is to keep opened just factories that are in used.

$T P F_{t}=\sum_{i} p f c_{i} \cdot\left(\sum_{t \prime \leq t} w_{i t}-\sum_{t \prime \leq t} w_{i t \prime}^{u}\right) \quad \forall t$

Total investment expansion costs in each period $T E_{t}$ are determined in Eq. (58). This investment cost $e c_{j}$ must be paid whenever an expansion is decided $\left(y_{j t}^{e}=1\right)$.

$T E_{t}=\sum_{j} e c_{j} \cdot y_{j t}^{e} \quad \forall t$

Total investment costs in each period for expansion of factories $T E P_{t}$ are determined in Eq. (59). This investment cost $e c p_{i}$ is considered in the period the expansion is decided ( $w_{i t}^{e}=1$ ).

$T E P_{t}=\sum_{i} e c p_{i} \cdot w_{i t}^{e} \quad \forall t$

If a warehouse is uninstalled, then the fixed costs $u c_{j}$ have to be paid. Total elimination cost in each period $\left(T U_{t}\right)$ is calculated in Eq. (60).

$T U_{t}=\sum_{j} u c_{j} \cdot y_{j t}^{u} \quad \forall t$ 
As for warehouses, when a factory is uninstalled there is a fixed costucp $p_{i}$ to be paid. Total elimination cost of factories in each period $\left(T U P_{t}\right)$ is calculated in Eq. (61).

$T U P_{t}=\sum_{i} u c p_{i} \cdot w_{i t}^{u} \quad \forall t$

Equation (62) determines the amount of total variable costs per period in the warehouses. It is calculated as the product of the unit variable $\operatorname{cost} g_{j}$, the daily demand $\mu_{i j k p t}^{\text {new }}$ and the number of days per period $\chi$.

$\operatorname{TOV}_{t}=\sum_{i} \sum_{j} \sum_{k} \sum_{p} g_{j} \cdot \mu_{i j k p t}^{n e w} \cdot \chi \quad \forall t$

Similarly, Eq. (63) determines the amount of total variable costs per period in factories. It is calculated as the product of the unit variable cost $g p_{i}$, the daily demand $\mu_{i j k p t}^{\text {new }}$ and the number of days per period $\chi$.

$T P V_{t}=\sum_{i} \sum_{j} \sum_{k} \sum_{p} g p_{i} \cdot \mu_{i j k p t}^{n e w} \cdot \chi \quad \forall t$

Repair cost in each period t, is given by Eq. (64). This cost is determined for standard and tailor made units.

$T R_{t}=\sum_{j \epsilon S C} \sum_{k} \sum_{p} g r_{j p} \cdot \mu_{j k p t}^{u s e d} \cdot \chi+\sum_{j \epsilon S C} \sum_{k} \sum_{s \epsilon K T_{k s}} g r^{\prime}{ }_{j s} \cdot \tau_{j k s t}^{u s e d} \cdot \chi \forall t$

Transportation costs from factories are determined in Eq. (65). Unit transportation cost from factories $i$ to warehouses $j c 1_{i j}$ are multiplied by standard daily demand $\mu_{i j k p t}^{\text {new }}$ and the number of days $\chi$. In the case of tailor made spare parts, unit transportation cost from factories $i$ to customer site $\mathrm{k}$ $c 3_{i k}$ is multiplied by the daily demand $\tau_{i k s t}^{\text {new }}$ and days per period $\chi$.

$\mathrm{TTF}_{t}=\sum_{i} \sum_{j \epsilon S C} \sum_{k} \sum_{p} c 1_{i j} \cdot \mu_{i j k p t}^{n e w} \cdot \chi+\sum_{i} \sum_{k} \sum_{s \epsilon K T_{k s}} c 3_{i k} \cdot \tau_{i k s t}^{n e w} \cdot \chi \forall t$

Transportation costs from warehouses are determined in Eq. (66). Unit transportation cost from warehouses $j$ to customers $k c 2_{j k}$ are multiplied by standard daily demand $\mu_{i j k p t}^{\text {new }}$ and the number of days $\chi$. In the case of repaired standard and tailor made spare parts, the same unit transportation cost is multiplied by 2 to consider the double route, from customers to workshops, and from workshops to customers. This cost is multiplied by the daily demand satisfied with repaired used spare parts ( $\tau_{j k s t}^{\text {used }}$ in the case of tailor made and $\mu_{j k p t}^{\text {used }}$ in the case of standard spare parts) and by the days per period $\chi$. $T T W_{t}=\sum_{i} \sum_{j} \sum_{k} \sum_{p} c 2_{j k} \cdot \mu_{i j k p t}^{n e w} \cdot \chi+\sum_{j \epsilon S C} \sum_{k} 2 \cdot c 2_{j k} \cdot \chi\left(\sum_{p} \mu_{j k p t}^{u s e d}+\sum_{s \in K T_{k s}} \tau_{j k s t}^{u s e d}\right) \forall t$

Mean inventory costs in warehouses are calculated in Eq. (67) as the unit inventory cost per day $\theta 1_{j p}$ multiplied by the daily demand $\mu_{i j k p t}^{\text {new }}$ and the processing time $t 1_{i j p}$.

$T P W_{t}=\sum_{i} \sum_{j} \sum_{k} \sum_{p} \theta 1_{j p} \cdot \mu_{i j k p t}^{n e w} \cdot t 1_{i j p} \quad \forall t$ 
Similarly, mean inventory costs at customer sites are calculated in Eq. (68)for special and tailor made units. This cost is determined multiplying the unit inventory cost per day, $\theta 2_{k p}$ and $\theta 3_{k p}$, multiplied by the daily demand, $\mu_{i j k p t}^{\text {new }}$ and $\tau_{i k s t}^{n e w}$, and the processing time, $t 2_{j k p}$ and $t 3_{i k s}$, respectively. $T P C_{t}=\sum_{i} \sum_{j} \sum_{k} \sum_{p} \theta 2_{k p} \cdot \mu_{i j k p t}^{n e w} \cdot t 2_{j k p}+\sum_{i} \sum_{k} \sum_{s \in K T_{k s}} \theta 3_{k p} \cdot \tau_{i k s t}^{n e w} \cdot t 3_{i k s} \forall t$

Safety stock costs are determined by Eq. (69). The first term indicates safety stock cost at warehouses for standard units, the second term calculates safety stock cost at customer sites for standard units, while the third term determines the safety stock cost at customer sites for tailor made parts.

$\operatorname{TSS}_{t}=$

$\sum_{j} \sum_{p} h 1_{j p} \cdot s s_{j p t}+\sum_{k} \sum_{s \notin K T_{k s}} \sum_{c \epsilon K S C_{k s c}} h 2_{k} \cdot \lambda 2_{k s} \cdot \sigma_{k s c t} \cdot \sqrt{l_{k s c t}}+\sum_{k} \sum_{s \epsilon K T_{k s}} \sum_{c \epsilon K S C_{k s c}} h 2_{k} \cdot$

$\lambda 2_{k s} \cdot \sigma_{k s c t} \cdot \sqrt{m_{k s c t}} \quad \forall t$

As it was explained in Section 3.5, lost sales costs are also included in the objective function. Equation (70) determines the lost sales cost for standard parts while Eq. (71) calculates this cost for tailor made units.

$$
\begin{aligned}
& T B T_{t}=\sum_{k} \sum_{s \notin K T_{k s}} \sum_{c \epsilon K S C_{k s c}} b 1_{k s} \cdot 0.45 \cdot \sigma_{k s c t} \cdot \sqrt{l_{k s c t}} \cdot e^{\lambda 2_{k s} /{ }_{-0.59} \cdot \chi \cdot \sum_{j} \frac{z_{j k t}}{t 2_{j k s}} \quad \forall t} \\
& T B S_{t}=\sum_{k} \sum_{s \in K T_{k s}} \sum_{c \epsilon K S C_{k s c}} b 1_{k s} \cdot 0.45 \cdot \sigma_{k s c t} \cdot \sqrt{m_{k s c t}} \cdot e^{\lambda 2_{k s} /{ }_{-0.59} \cdot \chi \cdot \sum_{i} \frac{u_{i k s t}}{t 3_{i k s}} \forall t}
\end{aligned}
$$

Including all these costs, the objective function is given by Eq. (72).

\section{Min C}

$C=\sum_{t} \frac{T I_{t}+T P I_{t}+T O F_{t}+T P F_{t}+T E_{t}+T E P_{t}+T U_{t}+T U P_{t}+T O V_{t}+T P V_{t}+T R_{t}+T T F_{t}+T T W_{t}+T P W_{t}+T P C_{t}+T S S_{t}+T B T_{t}+T B S_{t}}{(1+i r)^{t}}$

Finally, the original problem P0 is given by Eqs. (8)-(72). This is an MINLP formulation due to bilinear terms in Eqs. (24)and(30), and square root terms in Eqs.(31) and (69)-(71).

\subsection{Problem reformulation as an MILP}

In this section, non-linear equations from problem P0 are transformed to obtain a linear relaxation. In the case of bilinear term (products of binaries or continuous times binary variable) exact reformulations are proposed, while a linear approximation is used for the square root terms which yields a lower bound of the original functions.

Considering that $x_{i j p t}$ and $z_{j k t}$ are binary variables, the nonlinear term appearing in Eq. (24) can be replaced by a new variable $x z_{i j k p t}$ adding the following equations:

$$
\begin{aligned}
& x z_{i j k p t} \leq x_{i j p t} \quad \forall i, \forall j, \forall k, \forall p, \forall t \\
& x z_{i j k p t} \leq z_{j k t} \quad \forall i, \forall j, \forall k, \forall p, \forall t \\
& x z_{i j k p t} \geq z_{j k t}+x_{i j p t}-1 \quad \forall i, \forall j, \forall k, \forall p, \forall t
\end{aligned}
$$


where $0 \leq x z_{i j k p t} \leq 1$

Equation (24) can be now replaced by:

$\mu_{i j k p t}^{n e w} \leq \sum_{\substack{s \in P S_{p s} \\ s \notin C T_{k s}}} \sum_{c \in K S C_{k s c}} \mu_{k s c t} \cdot x Z_{i j k p t}$

$$
\forall i, \forall j, \forall k, \forall p, \forall t
$$

Similarly, the bilinear term from Eq. (30) involving continuous variable $s_{j p t}$ and binary variable $z_{j k t}$, can be replaced by a new variable $s z_{j k p t}$. Auxiliary variable $s z 1_{j k p t}$ is also introduced in the formulation, as follows:

$$
\begin{array}{ll}
s Z_{j k p t} \leq z_{j k t} \cdot s_{j p t}^{U P} & \forall j, \forall k, \forall p, \forall t \\
s Z_{j k p t} \leq\left(1-z_{j k t}\right) \cdot s_{j p t}^{U P} & \forall j, \forall k, \forall p, \forall t \\
s_{j p t} \geq s z_{j k p t}+s z 1_{j k p t} & \forall j, \forall k, \forall p, \forall t
\end{array}
$$

Eq. (30) is now replaced by (52):

$$
l_{k s c t} \geq \sum_{j} s z_{j k p t}+\sum_{j} t 2_{j k p} \cdot z_{j k t}-R_{k s c} \quad \forall i, \forall(k, s, c) \in K S C_{k s c}, \forall(k, s) \notin K T_{k s}, \forall t
$$

In the case of Eq. (31), since a bilinear term and a square root is involved, then the linearization is given in two steps. First the bilinear term of $z_{j k t} \cdot n_{j p t}$ is replaced by a new variable $n z_{j k p t}$. Also an auxiliary variable $n z_{j k p t}$ is added.

$$
\begin{array}{ll}
n z_{j k p t} \leq z_{j k t} \cdot n_{j p t}^{U P} & \forall j, \forall k, \forall p, \forall t \\
n z_{j k p t} \leq\left(1-z_{j k t}\right) \cdot n_{j p t}^{U P} & \forall j, \forall k, \forall p, \forall t \\
n_{j p t} \geq n z_{j k p t}+n z 1_{j k p t} & \forall j, \forall k, \forall p, \forall t
\end{array}
$$

Then, we define a new variable $n z v_{\text {jpt }}$ in order to transform the right hand side of Eq. (31) into an univariate square root term.

$$
n z v_{j p t}=\sum_{k} \sum_{\substack{s \in P S \\ s \notin C T}} \sum_{c \in K S C_{k s c}} \sigma_{k s c t}^{2} \cdot n z_{j k p t} \quad \forall j, \forall p, \forall t
$$

Now, Eq. (31) can be rewritten as a linear equation as follows:

$$
s s_{j p t}=\lambda_{j p} \cdot n z v_{j p t} / \sqrt{n z v_{j p t}^{U P}} \quad \forall j, \forall p, \forall t
$$

It is worth to note that Eq. (85) provides a lower bound of the original Eq. (31).

Eq. (69) calculates safety stock costs and can be reformulated applying also a lower bound. In this case, since in general the lower bound of the variables involved $\left(l_{k s c t}\right.$ and $\left.m_{k s c t}\right)$ is greater than zero, we can obtain a tighter approximation of the original equation, as proposed by Nyberg et al. (2013). 


$$
\begin{gathered}
T S S_{t}=\sum_{j} \sum_{p} h 1_{j p} \cdot s s_{j p t}+\sum_{k} \sum_{s \notin C T_{k s}} \sum_{c \in K S C_{k c s}} h 2_{j p} \cdot \lambda 2_{k s} \cdot \sigma_{k s c t} \cdot\left[l_{k s c t} \cdot\left(\frac{\sqrt{l_{k s c t}^{L O}}-\sqrt{l_{k s c t}^{U P}}}{l_{k s c t}^{L O}-l_{k s c t}^{U P}}\right)+\right. \\
\left.\sqrt{l_{k s c t}^{U P}}-l_{k s c t}^{U P} \cdot\left(\frac{\sqrt{l_{k s c t}^{L O}}-\sqrt{l_{k s c t}^{U P}}}{l_{k s c t}^{L O}-l_{k s c t}^{U P}}\right)\right]+\sum_{k} \sum_{s \in C T_{k s}} \sum_{c \in K S C_{k c s}} h 2_{j p} \cdot \lambda 2_{k s} \cdot \sigma_{k s c t} \cdot \\
{\left[m_{k s c t} \cdot\left(\frac{\sqrt{m_{k s c t}^{L O}}-\sqrt{l m_{k s c t}^{U P}}}{m_{k s c t}^{L O}-m_{k s c t}^{U P}}\right)+\sqrt{m_{k s c t}^{U P}}-m_{k s c t}^{U P} \cdot\left(\frac{\sqrt{m_{k s c t}^{L O}}-\sqrt{m_{k s c t}^{U P}}}{m_{k s c t}^{L O}-m_{k s c t}^{U P}}\right)\right] \quad \forall t}
\end{gathered}
$$

Regarding the square root in Eq. (70) we can regroup the summation over set $j$ as follows:

$$
\mathrm{TBS}_{t}=\sum_{k} \sum_{s} \sum_{c} b 1_{k s} \cdot \sigma_{k s c t} \cdot 0.45 \cdot e^{\lambda 2_{k s} /-0.59} \cdot \chi \cdot \sum_{j} \frac{\sqrt{l_{k s c t}} \cdot z_{j k t}}{t 2_{j k s}} \forall k, \forall(p, s) \in P S_{p s}, \forall c, \forall t
$$

Since $z_{j k t} \in\{0,1\}, \sqrt{l_{k s c t}} \cdot z_{j k t}=\sqrt{l_{k s c t} \cdot z_{j k t}}$, then we can introduce variable $l^{\prime}{ }_{j k s c t}$ to replace the bilinear product:

$$
\mathrm{TBS}_{t}=\sum_{k} \sum_{s} \sum_{c} b 1_{k s} \cdot \sigma_{k s c t} \cdot 0.45 \cdot e^{\lambda 2_{k s} /-0.59} \cdot \chi \cdot \sum_{j} \frac{\sqrt{l^{\prime}{ }_{j k s c t}}}{t 2_{j k s}} \forall k, \forall(p, s) \in P S_{p s}, \forall c, \forall t
$$

Note that since $\sum_{j} Z_{j k t}=1$ in Eq. (8), then:

$$
\begin{array}{lc}
\sum_{j} l^{\prime}{ }_{j k s c t}=l_{k s c t} & \forall k, \forall s, \forall c, \forall t \\
l^{\prime}{ }_{j k s c t} \geq l_{k s c t}^{L O} \cdot z_{j k t} & \forall k, \forall s, \forall c, \forall t \\
l^{\prime}{ }_{j k s c t} \leq l_{k s c t}^{U P} \cdot z_{j k t} & \forall k, \forall s, \forall c, \forall t
\end{array}
$$

Then, the same linear approximation applied to Eq. (70) can be now implemented for Eq. (71) as shown in Eq.(92):

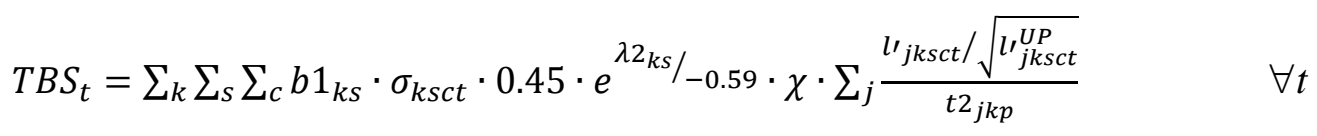

Similarly, for Eq. (71), variable $m_{i k s c t}^{\prime}$ can be introduced to replace the bilinear product of $m_{k s c t}$ by $u_{i k s t}$. Eqs. (93) - (95) are added to the formulation and Eq. (71) can be replaced by (96). Note that $\operatorname{since} \sum_{i} u_{i k s t}=1$ in Eq. (10), then:

$$
\begin{array}{cc}
\sum_{i} m_{i k s c t}^{\prime}=m_{k s c t} & \forall k, \forall s, \forall c, \forall t \\
m_{i k s c t}^{\prime} \geq m_{k s c t}^{L O} \cdot u_{i k s t} & \forall k, \forall s, \forall c, \forall t \\
m_{i k s c t}^{\prime} \leq m_{k s c t}^{U P} \cdot u_{i k s t} & \forall k, \forall s, \forall c, \forall t \\
T B S_{t}=\sum_{k} \sum_{s} \sum_{c} b 1_{k s} \cdot \sigma_{k s c t} \cdot 0.45 \cdot e^{\lambda 2_{k s} /-0.59 \cdot \chi \cdot \sum_{i} \frac{m^{\prime}{ }_{i k s c t} / \sqrt{m^{\prime U P}{ }_{j k s c t}^{U P}}}{t 3_{i k s}}} & \forall t
\end{array}
$$


Then, the MILP reformulation (P1) of the MINLP model (P0) is given by Eq. (8) - (23), (25)(29), (32) - (68), (72) - (86) and (89) - (96).

\section{Solution approach}

Due to the non-convex nature of P0, solving this MINLP formulation is not always straightforward. For that reason, we solve the problem applying a set of steps detailed by You and Grossmann (2010). First we solve the MILP model P1 which provides an initial lower bound to the original formulation. Next, we fix the integer variables of P1 into P0 obtaining P0'. This model is now a nonlinear programming (NLP) formulation which provides an upper bound to the original model. It is solved using P1 solution as initial values for the continuous variables. The solution obtained from P0' is used to provide a piece-wise linearization of the variables involved in the square root terms. The aim of this procedure is to find a tighter lower bound in each MILP iteration. The solution of this MILP is again used to formulate an NLP model and provide the initial values to the continuous variables. This procedure is repeated until the gap between the lower and upper bounds is sufficiently small.

\section{Results}

Three examples are presented in this section in order to illustrate the model formulation and approach proposed. These examples are executed in GAMS 23.7 using DICOPT for the MINLP models, CONOPT 3.14A for the NLP models and CPLEX 12.3 for the MILP models, over a CPU Intel Core i7, $3.40 \mathrm{GHz}$ with a $8 \mathrm{~GB}$ of RAM.

\subsection{Example 1: small supply chain}

In order to illustrate the problem, the first example is given by a small supply chain with two potential factories, three warehouses that can be also used as repair work-shops and six customers as shown in Figure 11. In this case, three units are considered and the horizon planning is given by three years.

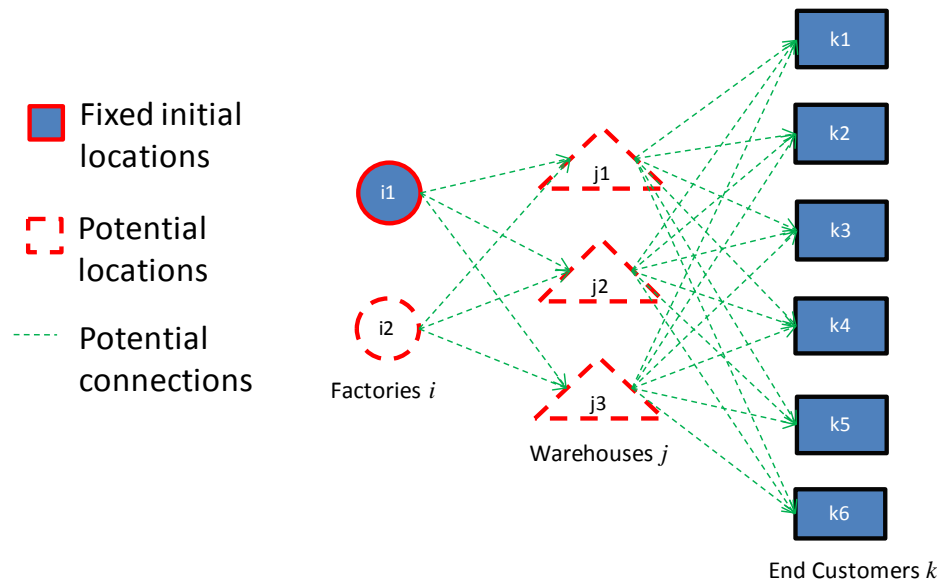

Figure 11 . Small supply chain example 
This example is solved applying the original MINLP formulation (P0) and the procedure explained in Section 5. The computational performance from the first approach is presented in Table 1, while the performance of the second is shown in Table 2. The last column of Table 2 shows the relative gap, between the upper bound (UB) given by the MILP model and the lower bound (LB) provided by the NLP, which decreases in each iteration. Comparing both alternatives, the proposed procedure is more efficient due to the final solution obtained. Even if CPU time is compared, the proposed approach achieves a better solution in the first iteration consuming 0.594 seconds. It is worth to mention that the MINLP model is initialized according to solution obtained in P1 (MILP 1 in Table 2).

Table 1. MINLP model performance for example 1

\begin{tabular}{lcccc}
\hline Objective Function & Equations & $\begin{array}{c}\text { Binary } \\
\text { Variables }\end{array}$ & $\begin{array}{c}\text { Continuous } \\
\text { Variables }\end{array}$ & CPUs \\
\hline \$19517593.7 $\left(^{*}\right)$ & 1974 & 368 & 917 & 2.08 \\
\hline$\left(^{*}\right)$ Integer gap $0.00 \%$ & & & &
\end{tabular}

Table 2. Proposed approach performance for example 1

\begin{tabular}{ccccccc}
\hline Iterations & Objective Function & Equations & Binary Variables & Continuous Variables & CPUs & (UB-LB)/UB \\
\hline MILP 1 & $\$ 19402074.4$ & 1974 & 368 & 917 & 0.500 & $0.17 \%$ \\
NLP 1 & $\$ 19434386.9$ & 815 & 0 & 541 & 0.094 & \\
MILP 2 & $\$ 19422685.7$ & 2406 & 530 & 1349 & 3.475 & $0.09 \%$ \\
NLP 2 & $\$ 19439604.4$ & 815 & 0 & 541 & 0.109 & 2.621 \\
MILP 3 & $\$ 19425657.0$ & 2514 & 584 & 1403 & $0.04 \%$ \\
NLP 3 & $\$ 19434138.6$ & 815 & 0 & 541 & 0.094 & \\
\hline
\end{tabular}

For the solution at $\$ 194,434,138.6$ from NLP 3, Figure 11 shows that factory i1 is already installed at the beginning of the horizon planning. However it can be uninstalled if necessary. According to the model solution from the last iteration of the proposed approach, the supply chain in period one and two is given by Figure 12. Note that factory i2 is installed in period 1, and the three potential warehouses are also selected in the same period. In period 2, factory i2 and warehouse j2 are expanded. No investment decision is made in period 3 and the connections between the nodes of the supply chain are the same as the ones in period 2. From Fig. 12, it is also shown that the warehouses $\mathrm{j} 1$ and $\mathrm{j} 2$ are also used as repair work-shop while $\mathrm{j} 3$ is dedicated to store and deliver new units. 

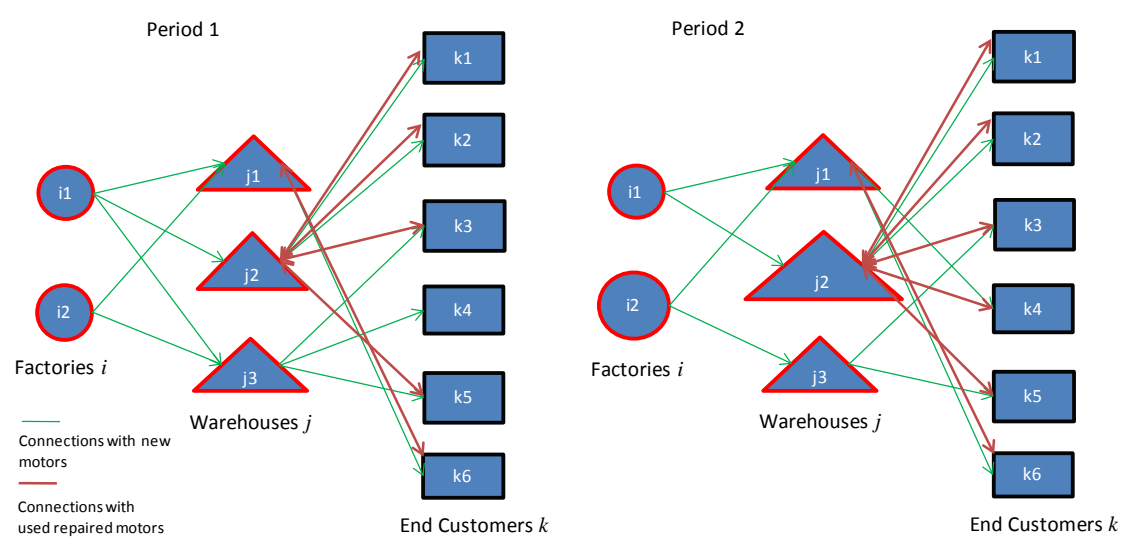

Figure 12. Supply chain in period 1 and 2 for example 1

Figures 13 and 14 show the capacity profiles for warehouses and factories, respectively, in the horizon planning. Safety stock required in warehouses is presented in Table 3.

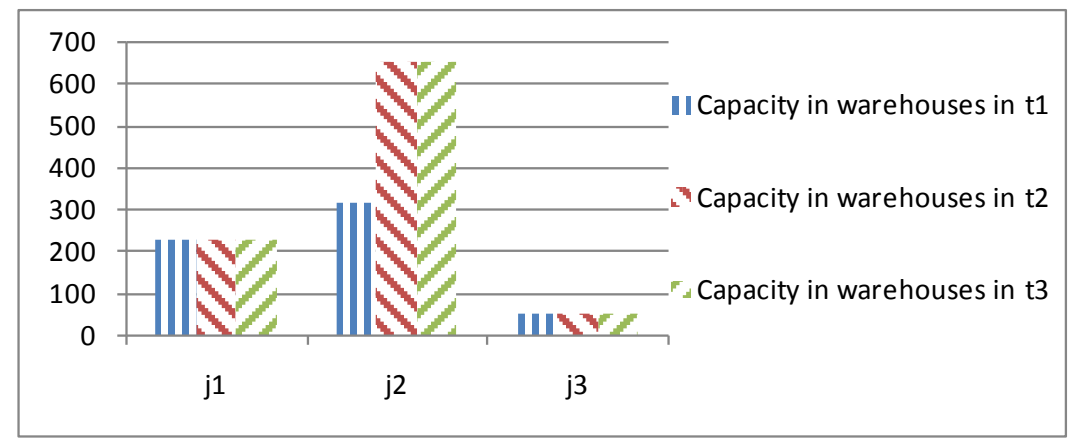

Figure 13. Capacity profile for warehouses

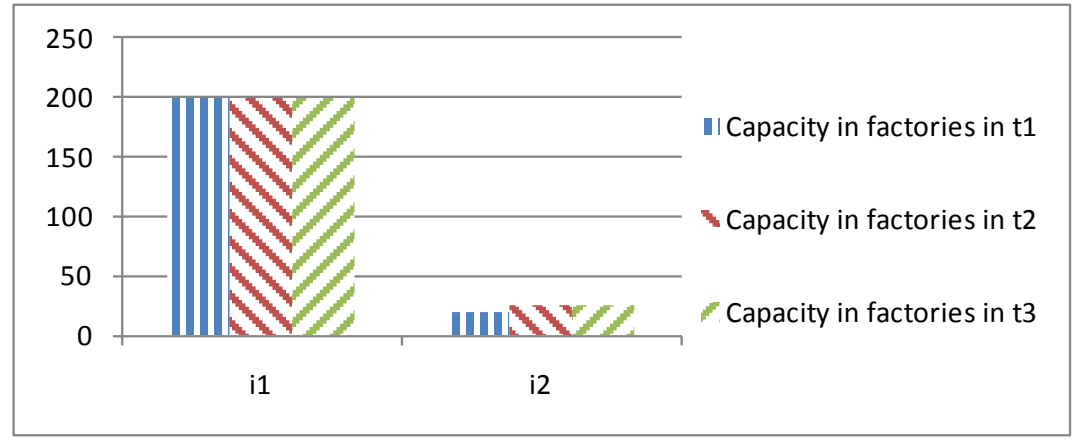

Figure 14. Capacity profile for factories

Table 3. Safety stock at warehouses for example 1

\begin{tabular}{ccccc}
\hline & & $\mathrm{t} 1$ & $\mathrm{t} 2$ & $\mathrm{t} 3$ \\
\hline & $\mathrm{p} 1$ & & 69.0 & 74.2 \\
$\mathrm{j} 1$ & $\mathrm{p} 2$ & & 55.0 & 62.7 \\
& $\mathrm{p} 3$ & 25.6 & 29.2 & 33.2 \\
\hline \multirow{2}{*}{ j2 } & $\mathrm{p} 1$ & & 58.8 & \\
& $\mathrm{p} 2$ & & 77.3 & \\
\hline & $\mathrm{p} 1$ & 6.7 & & \\
$\mathrm{j} 3$ & $\mathrm{p} 2$ & & & \\
& $\mathrm{p} 3$ & 16.2 & 35.0 & 39.6 \\
\hline & & & &
\end{tabular}




\subsection{Example 2: Larger supply chain}

This example considers a supply with four factories, ten warehouses, twenty customers, five standard units, ten special units and one tailor made units. Eight warehouses can be used as repair work-shops. The horizon planning is given by five time periods. The factories are installed at the beginning of the horizon planning, but they can be expanded or eliminated if this is convenient. Also three warehouses are already in operation but these decisions (expansion or elimination) can be made. The initial supply chain is shown in Figure 15.

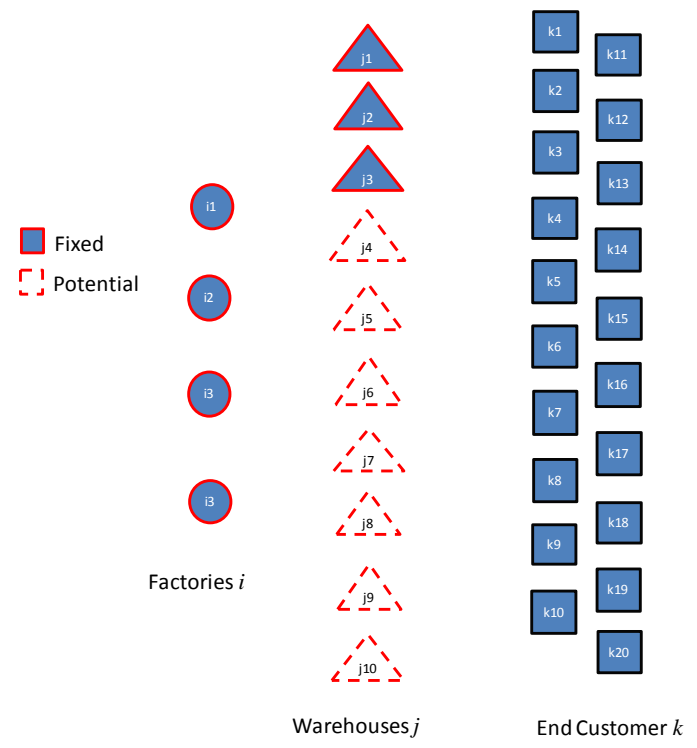

Figure 15. Initial supply chain for example 2

Table 4 shows the MINLP model (P0) size and performance where the stopping criterion is given by 10000 seconds of execution. The proposed approach presents better results as shown in Table 5 .

Table 4. MINLP model performance for example 2

\begin{tabular}{ccccc}
\hline Objective Function & Equations & $\begin{array}{c}\text { Binary } \\
\text { Variables }\end{array}$ & $\begin{array}{c}\text { Continuous } \\
\text { Variables }\end{array}$ & CPUs \\
\hline \$50243744.6 $\left({ }^{*}\right)$ & 113556 & 8843 & 57238 & 10000 \\
\hline$\left(^{*}\right)$ Integer gap $1.57 \%$ & & & &
\end{tabular}

Table 5. Proposed approach performance for example 2

\begin{tabular}{cccccccc}
\hline Iterations & $\begin{array}{c}\text { Objective } \\
\text { Function }\end{array}$ & $\begin{array}{c}\text { Integer } \\
\text { gap }\end{array}$ & Equations & $\begin{array}{c}\text { Binary } \\
\text { Variables }\end{array}$ & $\begin{array}{c}\text { Continuous } \\
\text { Variables }\end{array}$ & CPUs & (UB-LB)/UB \\
\hline MILP 1 & $\$ 48413413.3$ & $0.000 \%$ & 113556 & 8843 & 57238 & 12.710 & $0.45 \%$ \\
NLP 1 & $\$ 48632001.4$ & & 37318 & 0 & 22951 & 2.36 & \\
MILP 2 & $\$ 48591510.7$ & $0.298 \%$ & 119996 & 11258 & 65033 & 29.33 & $0.18 \%$ \\
NLP 2 & $\$ 48679436.9$ & & 37318 & 0 & 22951 & 12.36 & \\
MILP 3 & $\$ 48614142.9$ & $0.267 \%$ & 121606 & 12063 & 65838 & 57.99 & $0.03 \%$ \\
NLP 3 & $\$ 48627740.0$ & & 37318 & 0 & 22951 & 21.84 & \\
\hline
\end{tabular}


Figure 16 shows the supply chain design in periods one and two. In the first period, warehouses j6, j8 and j9 are installed. The first one is not used to store new units. In period two, factory i2 is expanded and i4 is uninstalled. Now factory i2 can serve more warehouses due to the increase in the capacity.
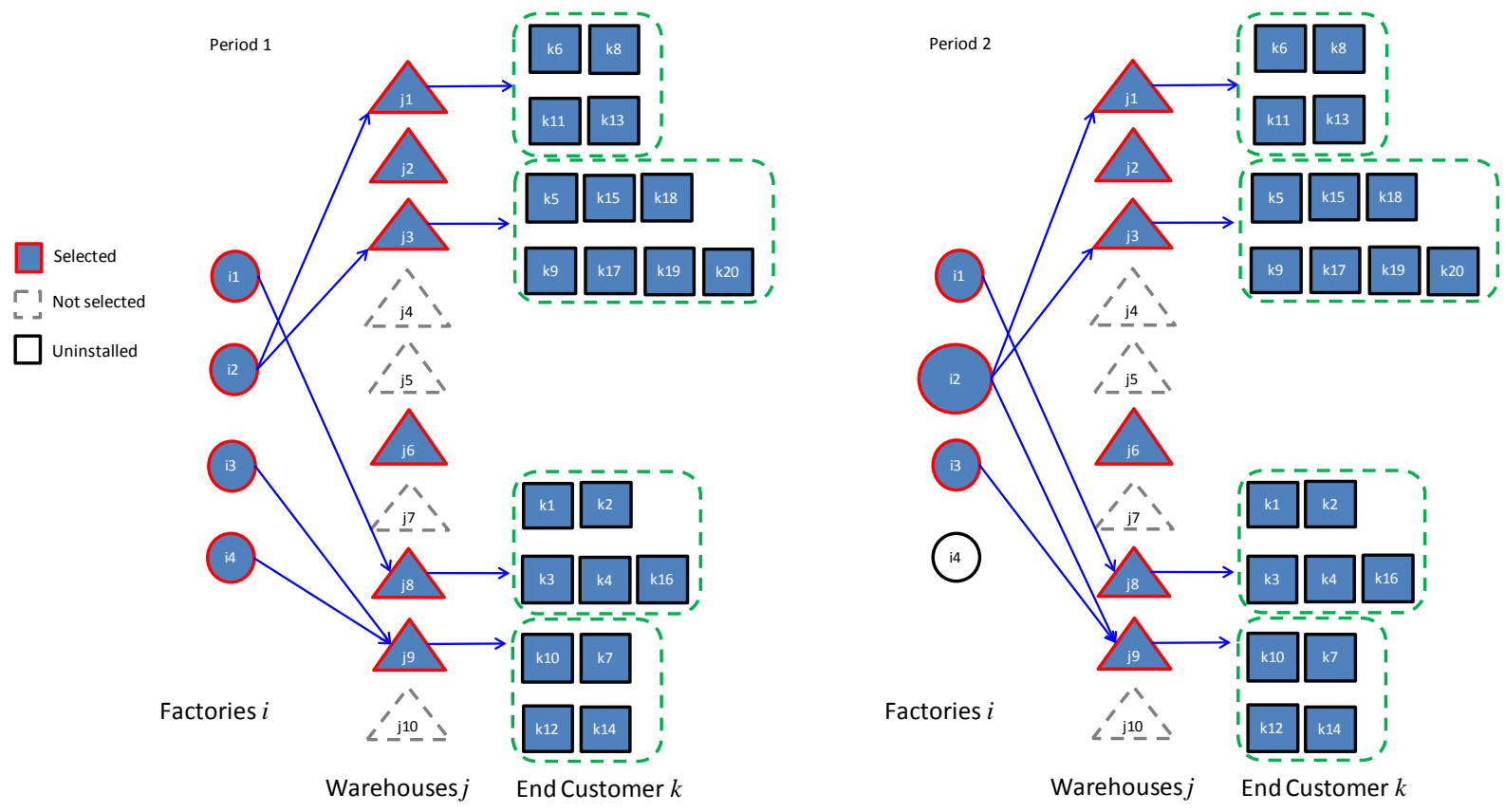

Figure 16. Supply chain configuration for new units in periods 1 and 2 for example 2

The links between the warehouses and customers remains in the second period as in the first one. Regarding used repaired units, j2 and j6 are dedicated to this activity. The connections between these shops and the end customers in period one are shown in Figure 17. The safety stock required in the warehouses is presented in Table 6. 


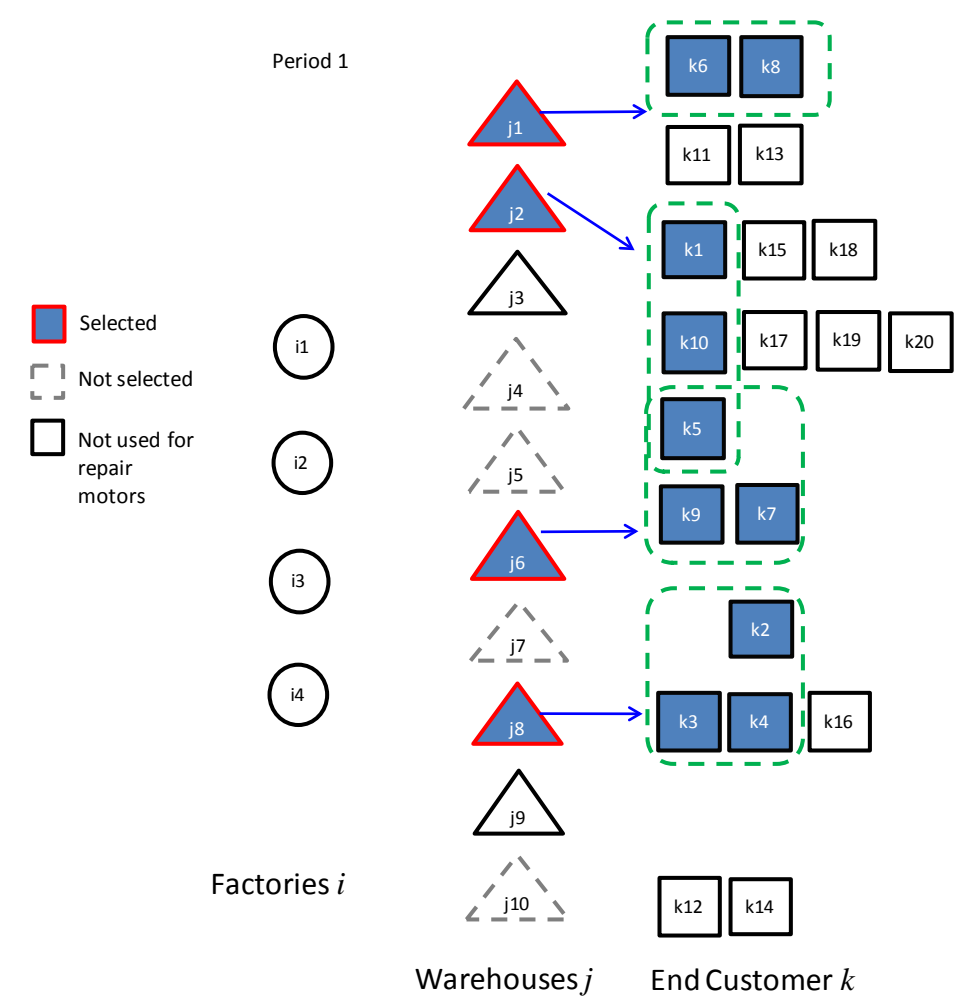

Figure 16. Supply chain configuration for used repaired units in period 1 for example 2

Table 6. Safety stock at warehouses for example 2

\begin{tabular}{ccccccc} 
& & $\mathrm{t} 1$ & $\mathrm{t} 2$ & $\mathrm{t} 3$ & $\mathrm{t} 4$ & $\mathrm{t}$ \\
\hline \multirow{4}{*}{$\mathrm{j} 1$} & $\mathrm{p} 1$ & 20.5 & 20.9 & 33.0 & 29.4 & 28.8 \\
& p2 & 35.7 & 36.4 & 51.6 & 44.6 & 44.7 \\
& $\mathrm{p} 3$ & 21.0 & 21.6 & 37.0 & 30.0 & 35.7 \\
& p4 & 52.8 & 54.5 & 69.5 & 67.4 & 68.2 \\
& p5 & 24.5 & 25.0 & & 39.6 & 33.2 \\
\hline \multirow{4}{*}{ j3 } & p2 & 32.8 & 33.2 & 48.4 & 47.4 & 51.6 \\
& p3 & 34.5 & 34.2 & 55.6 & 51.8 & 56.8 \\
& p4 & 47.6 & 48.6 & 72.4 & 65.6 & 76.5 \\
& p5 & 46.6 & 48.6 & 68.7 & 65.1 & 72.5 \\
\hline & p1 & 25.8 & 27.0 & & & 15.9 \\
& p2 & 36.2 & 37.5 & 59.0 & & 46.1 \\
j8 & p3 & 22.5 & 23.7 & 36.1 & 37.2 & 31.1 \\
& p4 & 46.8 & 48.2 & 6.6 & 69.0 & 56.2 \\
& p5 & 32.2 & 32.8 & 49.3 & 47.0 & 41.4 \\
\hline
\end{tabular}

\subsection{Example 3: Electric motors supply chain case study}

This case study considers the supply chain of electric motors over a five-year planning horizon. The data has been generated for testing the applicability of the optimization on a real-life test case. In this example case, all installed and potential warehouses are located in Sweden, while factories are located in Europe and Asia. The initial supply chain is given by seven factories, one warehouse, 
which is not a repair workshop (j1) and 27 customers $(k)$. No investments in new factories are allowed and lost sales costs are disregarded. Four additional warehouses can be installed in any of the five periods, and three of them can be repair workshops. A demand for 50 different motor types is included in the model.

In this case, due to the large model size, the problem is solved applying the MILP model P1 which provides a lower bound to the original MINLP. The solution indicates that two new warehouses $(\mathrm{j} 3$ and j4) are installed in period 1 as shown in Figure 17. The left side of Figure 17 shows the connections in the supply chain nodes for new motors, while the right side shows the links between repair work-shops and end customers for used motors. In this solution, one of the warehouses is only used for storage of new motors, while the other is exclusively used as a repair workshop. No expansions nor eliminations are implemented.

The performance and size of this model is presented in Table 7. The termination is given by the solver after 10000 sec of execution with a $1.73 \%$ optimality gap. The safety stock required at the warehouses is shown in Table 8.
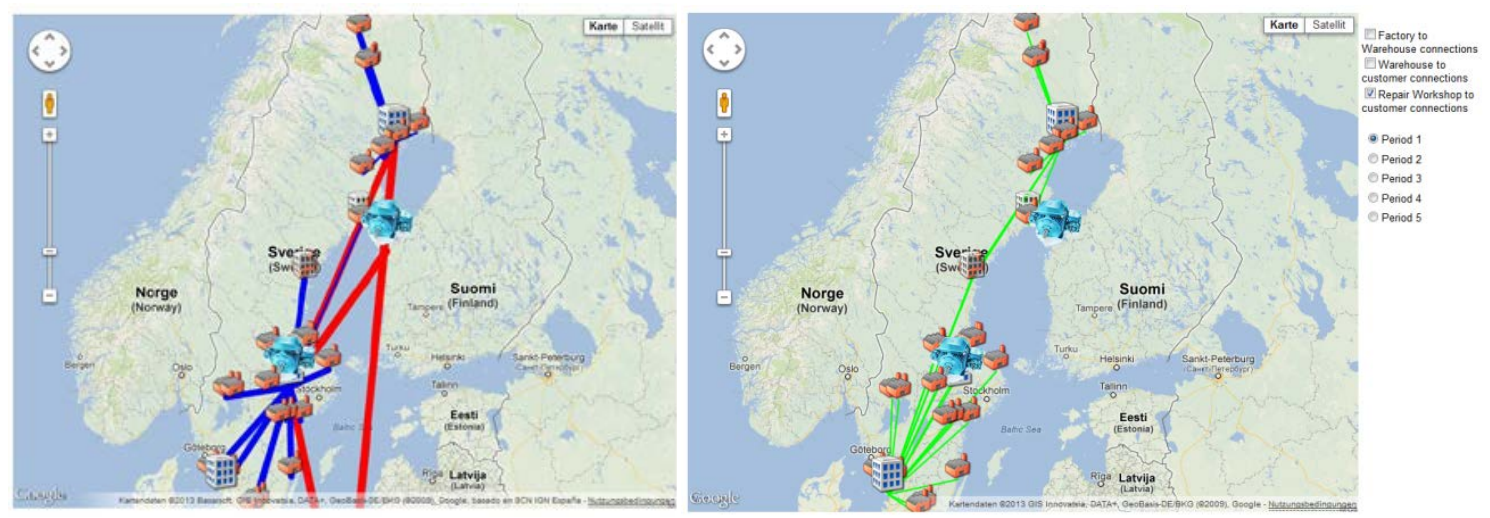

Figure 17. Supply chain design of example 3

Table 7. MILP model performance and objective function in example 3

\begin{tabular}{ccccc}
\hline $\begin{array}{c}\text { Objective } \\
\text { Function }\end{array}$ & $\begin{array}{c}\text { CPU time } \\
\text { (integer gap 1.73\%) }\end{array}$ & Equations & $\begin{array}{c}\text { Positive } \\
\text { Variables }\end{array}$ & $\begin{array}{c}\text { Binary } \\
\text { Variables }\end{array}$ \\
\hline$\$ 16881715.9$ & $10000 \mathrm{~s}$ & 212407 & 115064 & 12639 \\
\hline
\end{tabular}

Table 8. Safety stock at warehouses in example 3

\begin{tabular}{|c|c|c|c|c|c|c|}
\hline \multirow[b]{2}{*}{ Warehouse } & \multicolumn{6}{|c|}{ Motor } \\
\hline & ID & $\mathrm{t} 1$ & t2 & t3 & t4 & t5 \\
\hline \multirow[b]{3}{*}{ J1 } & P1 & 1.812 & 2.066 & 2.066 & 2.066 & 2.167 \\
\hline & $\mathrm{P} 2$ & 2.030 & 2.316 & 2.316 & 2.316 & 2.429 \\
\hline & P3 & 1.737 & 1.980 & 1.980 & 1.980 & 2.077 \\
\hline \multirow{6}{*}{ J1 } & $\mathrm{P} 4$ & 2.689 & 3.066 & 3.066 & 3.066 & 3.216 \\
\hline & P5 & 2.082 & 2.375 & 2.375 & 2.375 & 2.491 \\
\hline & $\mathrm{P} 1$ & 0.501 & 0.572 & 0.572 & 0.572 & 0.600 \\
\hline & $\mathrm{P} 2$ & 0.562 & 0.641 & 0.641 & 0.641 & 0.672 \\
\hline & P3 & 0.542 & 0.619 & 0.619 & 0.619 & 0.649 \\
\hline & $\mathrm{P} 4$ & 0.873 & 0.996 & 0.996 & 0.996 & 1.044 \\
\hline J3 & P5 & 0.574 & 0.655 & 0.655 & 0.655 & 0.687 \\
\hline
\end{tabular}




\section{Conclusions}

We have developed an MINLP model to determine the optimal supply chain structure over a multiperiod horizon planning considering demand uncertainty. Network decisions include the selection of new locations and the links that connect the different nodes in the supply chain in each period. Special characteristics from the electric motors industry are considered such as the demand of failing units that are at customer plants, and how this demand can be satisfied with new or used spare parts by the company. However, this model is generic and can also be applied to other type of industries. Model decisions such as new investment, capacity expansion and elimination of assets allow not only the design, but also the evaluation and re-design of a supply chain that is already in operation. From the inventory management perspective, safety stock, mean stock levels, capacity constraints and lost sales costs are also taken into account to satisfy customer orders according to the company commitments. Part II of this paper presents a decomposition approach in order to solve larger instances as well as to obtain a lower gap in reasonable computational time.

\section{Acknowledgments}

The authors gratefully acknowledge financial support for ABB through the Center for Advanced Process Decision-making, CONICET and Universidad Tecnologica Nacional.

\section{Nomenclature}

\section{Sets}

$c$ Criticality levels of motors

i Factories

$j$ Warehouses

$k$ End customers

$p$ Standard units

$s$ Special units

$t$ Time periods

$C T_{k s}$ Customers $k$ that allow used repaired units to satisfy their demand of units

$J F$ Subset of warehouses $j$ that are already installed (fixed) at the beginning of the horizon planning

$K S C_{k s c}$ Customers $k$ that order special units $s$ of criticality $c$

$K T_{k s}$ Customers $k$ that order tailor made units $s$

$P S_{p s}$ Special units $s$ belonging to standard unit $p$

SC Subset of warehouses $j$ that can be also considered as repair workshops

\section{Binary variables}

$u_{i k s t}$ if factory $i$ produces and delivers tailor made unit $s$ to end customer $k$ in period $t$ 
$v_{j k s t}$ if repair workshop $j$ repairs special units $s$ from customer $k$ in period $t$

$w_{i t}$ if factory $i$ is installed in period $t$

$w_{i t}^{e}$ if warehouse $j$ is expanded in period $t$

$w_{i t}^{u}$ if factory $i$ is uninstalled (eliminated) in period $t$

$x_{i j p t}$ if factory $i$ produces and delivers standard units $p$ to warehouse $j$ in period $t$

$y_{j t}$ if warehouse $j$ is installed in period $t$

$y_{j t}^{e}$ if warehouse $j$ is expanded in period $t$

$y_{j t}^{u}$ if warehouse $j$ is uninstalled (eliminated) in period $t$

$Z_{j k t}$ if warehouse $j$ delivers units to customer $k$ in period $t$

\section{Positive variables}

$c e_{j t}$ capacity expansion of warehouse $j$ in period $t$

$c e f_{i t}$ capacity expansion of factory $i$ in period $t$

$l_{\text {ksct }}$ net lead time of customer $k$ for special unit $s$ of criticality $c$ in period $t$

$l^{\prime}{ }_{j k s c t}$ net lead time of customer $k$ if special unit $s$ of criticality $c$ is provided by warehouse $j$ in period $t$

$m_{k s c t}$ net lead time of customer $k$ for tailor made unit $s$ of criticality $c$ in period $t$

$m_{i k s c t}^{\prime}$ net lead time of customer $k$ if tailor made unit $s$ of criticality $c$ is provided by warehouse $j$ in period $t$

$n_{j p t}$ net lead time of warehouse $j$ for standard unit $p$ in period $t$

$q_{j t}$ capacity of warehouse $j$ in period $t$

$q f_{i t}$ capacity of factory $i$ in period $t$

$s_{j p t}$ guaranteed service time of warehouse $j$ to its successive nodes in the supply chain for standard unit $p$ in period $t$

$S S_{j p t}$ safety stock of warehouse $j$ for standard unit $p$ in period $t$

$u c_{j t}$ capacity eliminated from warehouse $j$ in period $t$ (when the warehouse is uninstalled)

$u c f_{i t}$ capacity eliminated from factory $i$ in period $t$ (when the factory is uninstalled)

$\mu_{i j k p t}^{\text {new }}$ amount of demand of standard units $p$ from customer $k$ satisfied with new units from factory $i$ and warehouse $j$

$\mu_{j k s t}^{u s e d}$ amount of demand of special units $s$ from customer $k$ satisfied with used units from repair workshop $j$

$\tau_{i k s t}^{\text {new }}$ amount of demand of tailor made units $p$ from customer $k$ satisfied with new units from factory $i$

$\tau_{j k s t}^{u s e d}$ amount of demand of tailor made units $s$ from customer $k$ satisfied with used units from repair workshop $j$

\section{Parameters}

$b 1_{k s}$ unit annual lost sales cost for special unit $s$ at customer $k$

$c 1_{i j}$ unit transportation cost from factory $i$ to warehouse $j$ 
$c 2{ }_{j k}$ unit transportation cost from warehouse $j$ to customer $k$

$c 3_{i k}$ unit transportation cost from factory $i$ to customer $k$

$d_{k s}$ Fraction of demand that is back ordered or lost (customer disservice) for special unit $s$ from end customer $k$

$e c_{j}$ expansion investment cost for warehouse $j$
$e c p_{i}$ expansion investment cost for factory $i$
$f_{j}$ investment cost for installing warehouse $j$

$f p_{i}$ investment cost for installing factory $i$

$g_{j}$ variable handling cost of warehouse $j$

$G I_{\text {is }}$ guaranteed service time of factory $i$ for tailor made unit $s$

$g p_{i}$ variable production cost of factory $i$

$g r_{j p}$ variable repairing cost of repair workshop $j$ for standard unit $p$

$g r^{\prime}{ }_{j s}$ variable repairing cost of repair workshop $j$ for tailor made unit $s$

$h 1_{j p}$ unit safety stock cost for standard unit $p$ in warehouse $j$

$h 2_{k}$ unit safety stock cost at customer $k$

$I C_{j}$ initial capacity of warehouse $j$

$I C F_{i}$ initial capacity of factory $i$

ir interest rate

$o f c_{j}$ operational fixed cost for warehouse $j$

$p f c_{i}$ operational fixed cost for factory $i$

$Q_{k s}$ Reorder quantity for special unit s from end customer $k$

$Q D C_{j}^{U P}$ maximal capacity expansion in each period for warehouse $j$

$Q P_{i}^{U P}$ capacity of factory $i$

$R_{k s c}$ guaranteed service time expected by customer $k$ for special unit $s$ of criticality c

$r p_{k s}$ repairing probability of special unit $s$ from customer $k$

$S I_{i p}$ guaranteed service time of factory $i$ for standard unit $p$

$t 1_{i j p}$ order processing time of warehouse $j$ for standard unit $p$ if it is served by plant $i$, including material handling time in $j$, transportation time from plant $i$ to $j$, and inventory review period in the warehouse

$t 2_{j k p}$ order processing time of customer $k$ for standard unit $p$ if it is served by warehouse $j$, including material handling time in $k$, transportation time from warehouse $j$ to $k$, and inventory review period in the customer site

$t 3_{i k s}$ order processing time of customer $k$ for tailor made unit $s$ if it is served by plant $i$, including material handling time in $k$, transportation time from plant $i$ to $k$, and inventory review period in the customer site 
$t s u_{j p}$ average time that used standard units $p$ are kept in storage in warehouse $j$

$t t u_{j s}$ average time that used tailor made units $s$ are kept in storage in warehouse $j$

$u c_{j}$ investment cost if warehouse $j$ is uninstalled

${ }^{u c p_{i}}{ }_{\text {investment cost if factory } i \text { is uninstalled }}$

$\alpha_{p}$ production factor rate for standard unit $p$

$\beta_{p}$ size factor for standard unit $p$

$\beta 2_{\text {s }}$ size factory for special unit $s$

$\lambda_{j p}$ safety factor of warehouse $j$ for standard unit $p$

$\lambda 2_{k s}$ safety factor of customer $k$ for special unit $s$

$\mu_{\text {ksct }}$ mean demand of special units $s$ of criticality $c$ from customer $k$ in period $t$

$\theta 1_{j p}$ unit inventory cost for standard unit $p$ in warehouse $j$

$\theta 2_{k p}$ unit inventory cost for standard unit $p$ in customer $k$

$\sigma_{k s c t}$ demand standard deviation of special units $s$ of criticality $c$ from customer $k$ in period $t$

$\chi$ days in the year

\section{References}

Bossert, J.M. and Willems, S.P. A periodic-review modeling approach for guaranteed service supply chains. Interfaces. 2007, 37, 5, 420-435.

Charnes, A. and Cooper, W.W. Deterministic equivalents for optimizing and satisfying under chance constraints. Operations Research. 1963, 11, 18-39.

Daskin, M.S., Coullard, C.R. and Shen, Z.J.M. An Inventory-Location Model: Formulation, Solution Algorithm and Computational Results. Annals of Operations Research 110, 83-106, 2002.Kluwer Academic Publishers. The Netherlands.

Eppen, G.D. Effect of centralization on expected costs in a multi-location newsboy problem. Management Science, 1979, 25, 5, 498-501.

Grossmann, I.E. Enterprise-wide Optimization: A New Frontier in Process Systems Engineering. AIChE Journal, 2005, 51, 7, 1846-1857.

Gupta, A. and Maranas, C.D. Managing demand uncertainty in supply chain planning. Computers and Chemical Engineering. 2003, 27, 1219-1227.

Little, J.D.C. A proof of the queueing formula $L=\lambda$ W. Operation Research. 1961, 9, 383-387.

Nyberg, A., Grossmann, I.E. and Westerlund, T. An Efficient Reformulation of the Multiechelon Stochastic Inventory System with Uncertain Demands. AIChE Journal. 2013, 59, 23-28.

Parker, L.L. Economical reorder quantities and reorder points with uncertain demand. Naval Research Logistics Quarterly. 1964, 11, 4, 351-358.

Rodriguez, M.A., Vecchietti, A. Multicriteria optimization model for supply process problem under provision and demand uncertainty. Industrial and Engineering Chemistry Research. 2011, 50, 10630-10642. 
Sahinidis, N.V. Optimization under uncertainty: state-of-the-art and opportunities. Computers and Chemical Engineering. 2004, 28, 971-983.

Santoso, T., Ahmed, S., Goetschalckx, M. and Shapiro A. A stochastic programming approach for supply chain network design under uncertainty. European Journal of Operational Research. 2005, 167, 96-115.

Shen, S.J.M., Coullard, C. and Daskin, M.S. A Joint Location-Inventory Model. Transportation Science. 2003, 37, 1, 40-55.

You, F. and Grossmann, I.E. Design of responsive supply chains under demand uncertainty. Computers and Chemical Engineering. 2008, 32, 3090-3111.

You, F. and Grossmann, I.E. Integrated Multi-Echelon Supply Chain Design with Inventories Under Uncertainty: MINLP Models, Computational Strategies. AIChE Journal, 2010, 56, 2, 419-440.

Zipkin, P.H. Foundations of Inventory Management. Boston, MA. McGraw-Hill, 2000. 